Nouvelles perspectives en sciences sociales

Revue internationale de systémique complexe et d'études relationnelles

\title{
Communication, cognition et créativité dans les sociétés de la connaissance. À propos de deux dilemmes
}

\section{Bernard Ancori}

Volume 9, numéro 2, mai 2014

Sur le thème de l'échange

URI : https://id.erudit.org/iderudit/1025971ar

DOI : https://doi.org/10.7202/1025971ar

Aller au sommaire du numéro

\section{Éditeur(s)}

Prise de parole

ISSN

1712-8307 (imprimé)

1918-7475 (numérique)

\section{Découvrir la revue}

Citer cet article

Ancori, B. (2014). Communication, cognition et créativité dans les sociétés de la connaissance. À propos de deux dilemmes. Nouvelles perspectives en sciences sociales, 9(2), 45-93. https://doi.org/10.7202/1025971ar

\section{Résumé de l'article}

L'histoire des sciences et l'analyse bibliométrique suggèrent que les sciences de la nature, les sciences de l'ingénieur et, dans une moindre mesure, les sciences médicales seraient entrées depuis une trentaine d'années dans un régime d'équilibre stationnaire. Le but de cet article est d'identifier l'une des causes possibles d'une telle situation, et de proposer une solution efficace pour y remédier. Cette cause pourrait en effet tenir au privilège conféré, depuis le début des années 1970 et surtout depuis le milieu des années 1990, à la communication sociale au détriment de la cognition individuelle ou collective. Quant à la solution efficace pour y remédier, elle consisterait à développer systématiquement dans certains domaines des collaborations de recherche entre le monde académique et les mondes non académiques. Pour le montrer, nous procédons en deux étapes. La première met en évidence l'existence de deux dilemmes liés en ce qui concerne la structure et l'évolution d'un réseau sociocognitif complexe d'acteurs individuels : le dilemme entre créativité globale du réseau et proximités individuelles des acteurs, et celui qui se pose à chacun de ces derniers, dans chaque état du réseau, entre communiquer et cogiter. La seconde étape résume rapidement l'évolution des sciences et des technologies occidentales vers une techno-science dont la créativité semble s'essouffler, puis montre que la généralisation de forums hybrides pourrait redonner un surcroît de vigueur à cette créativité aujourd'hui atone. 


\title{
Communication, cognition et créativité dans les sociétés de la connaissance À propos de deux dilemmes
}

\author{
Bernard AnCori \\ Université de Strasbourg
}

D ans nos sociétés fondées sur la connaissance, les savoirs sont distribués et situés, au sein du monde académique, mais également parmi ce qu'il est convenu d'appeler les "profanes ». Ces sociétés se veulent innovatrices, et cherchent à exploiter les gisements de créativité existant parmi l'ensemble des acteurs, ainsi qu'à susciter l'émergence de gisements inédits. Dans le monde académique, il est souvent souligné que l'innovation scientifique et technique naît aux interfaces entre disciplines, d'où un appel récurrent à développer l'interdisciplinarité dans nos systèmes d'enseignement supérieur et de recherche ${ }^{1}$. La rencontre de chercheurs issus de champs scientifiques différents est à l'origine de fertilisations croisées de leurs savoirs, et de chocs entre ces deux zones de sens qu'Arthur Koestler appelait deux matrices sans relations antérieures ${ }^{2}$. Ces chocs peuvent alors

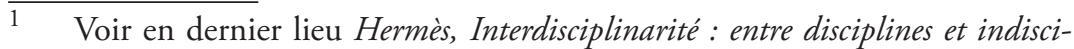
pline, $\mathrm{n}^{\circ}$ 67, Paris, CNRS Éditions, 2013, et notamment les contributions de Jean-Michel Besnier, "Seul le désordre est créateur. Pour en finir avec les bataillons disciplinaires ", p. 25-31, ainsi que de Sylvie Catellin et Laurent Loty, "Sérendipité et indisciplinarité ", p. 32-40.

2 Arthur Koestler, The Act of Creation, New York, Penguin Books, 1964. 
produire des analogies ou des métaphores inédites, créatrices de catégories mentales nouvelles dans les univers cognitifs des acteurs $^{3}$, et ils provoquent parfois des bouleversements épistémologiques majeurs ${ }^{4}$, sources potentielles de croissance économique ${ }^{5}$.

Néanmoins, le geste créateur n'est jamais qu'une condition nécessaire à la production de l'innovation : la synthèse intuitive qu'il produit, fût-elle élaborée, objectivée et rendue accessible par une œuvre, doit en outre être reçue, acceptée et intégrée par la culture correspondante ${ }^{6}$. La condition suffisante de la production d'innovation consiste donc en une forme d'acceptabilité culturelle qui en autorise la réception et l'appropriation, dont l'histoire montre qu'elle n'a rien de rapide 7 . La dialectique à l'œuvre lors de cette séquence longue entre le geste créatif et sa pleine réalisation englobe ainsi la genèse, la circulation, la réception et l'appropriation de connaissances nouvelles, et ceci à un niveau collectif aussi bien qu'individuel.

C'est dans le cadre de cette dialectique que nous voudrions mettre en évidence deux dilemmes concernant la créativité individuelle et collective d'acteurs considérés sous l'angle de ces activités cognitives et sociales très précises que sont respectivement la catégorisation issue de l'analogie (ou de la métaphore) et la communication interindividuelle. S'agissant de nos sociétés, une telle analyse n'a aucune raison de se limiter au seul champ

3 Bernard Ancori, "Analogie, évolution scientifique et réseaux complexes ", Nouvelles perspectives en sciences sociales. Revue internationale de systémique complexe et d'études relationnelles, vol. 1, n 1, 2005, p. 5-61.

4 Bernard Ancori, "Espace-temps d'un réseau sociocognitif complexe. II. Temporalités historiques et entropie sociocognitive ", Nouvelles perspectives en sciences sociales. Revue internationale de systémique complexe et d'études relationnelles, vol. 4, $\mathrm{n}^{\circ}$ 1, 2008, p. 9-76.

5 Bernard Ancori, "Analogie, métaphore et mutation féodale ", dans Sylvain David, Janusz Przychodzen et François-Emmanuel Boucher (dir.), Que peut la métaphore? Histoire, savoir et poétique, Paris, L'Harmattan, 2009, p. 115-131.

6 Judith Schlanger, "La pensée inventive », dans Judith Schlanger et Isabelle Stengers, Les concepts scientifiques : invention et pouvoir, Paris, La Découverte, 1989, p. 67-100.

7 Joël de Rosnay, "L'impact des nouvelles technologies dans l'échange de savoir ", dans Savoir échanger les savoirs, Paris, Éditions Textuel, 1997, p. $55-74$. 
du monde académique institué, car nombre de percées intellectuelles ont été opérées à sa marge - voire contre les institutions qui le structurent - qu'elles ont parfois réussi à étendre lorsqu'elles furent ensuite reconnues ${ }^{8}$. Mais nous dépasserons en outre ce champ ainsi élargi pour envisager également les savoirs " profanes ", et ceci pour trois raisons.

D'abord, pour répondre au caractère distribué et situé du savoir dans nos sociétés, souligné ici d'emblée : il est devenu difficile de continuer à poser l'existence d'une hiérarchie unique de connaissances, au sommet de laquelle trônerait un nouveau clergé de savants dominant une masse informe d'ignorants; en conséquence, de nombreuses voix appellent aujourd'hui à une vision plus horizontale de la distribution des savoirs et à la mobilisation de tous dans notre commune aventure d'exploration du réel ${ }^{9}$. Étendre notre domaine d'investigations aux savoirs profanes est alors d'autant plus pertinent que la fécondité potentielle de la fertilisation croisée évoquée plus haut est indexée sur la différence des univers cognitifs et sociaux ainsi confrontés, et il en va de même pour la créativité de l'analogie ou de la métaphore, d'autant plus puissante qu'est grande la tension entre le sujet primaire (mal compris) et le sujet secondaire (mieux compris) que rassemblent ces deux tropes afin d'éclairer la compréhension du premier de ces deux sujets par celle du second ${ }^{10}$.

Une deuxième raison de situer acteurs académiques et non académiques sur le même plan tient au fait que les processus cognitifs comme les régimes d'argumentation sont similaires chez

Geoffroy de Lagasnerie, Logique de la création. Sur l'Université, la vie intellectuelle et les conditions de l'innovation, Paris, Fayard, 2011.

9 Cf. Matthieu Calame, Lettre ouverte aux scientistes. Alternatives démocratiques à une idéologie cléricale, Paris, Éditions Charles Léopold Mayer, 2011, ou encore Marc Lipinski, Les sciences, un enjeu citoyen. Une politique écologiste de la recherche et de l'innovation, Paris, Éditions Les petits matins, 2011.

10 Ivor A. Richards, The Philosophy of Rhetoric, Oxford, Oxford University Press, 1936; Max Black, Models and Metaphors. Studies in Language and Philosophy, Ithaca, Cornell University Press, 1962; Max Black, " More about Metaphor ", dans Andrew Ortony (dir.), Metaphor and Thought, Cambridge, Cambridge University Press, 1963, p. 19-41; Arthur I. Miller, Insights of Genius. Imagery and Creativity in Science and Art, New York, Springer Verlag Inc., 1996. 
les uns et les autres. Ainsi, les modes de révision des croyances sont semblables lors de la formation de représentations scientifiques ou non scientifiques : ni les unes ni les autres n'ont de fondement certain, mais résultent d'un réarrangement de croyances précédentes face à de nouvelles informations ${ }^{11}$. Ce type de réarrangement est tel qu'Homo academicus et Homo vulgaris accordent identiquement une priorité aux informations nouvelles tout en manifestant un souci de conservatisme et de mémoire, et ceci avec un dosage qui dépend du contexte ${ }^{12}$. Quant aux régimes d'argumentation, ils font ici et là souvent appel aux mêmes figures de rhétorique : omniprésentes dans les processus cognitifs de tout acteur ${ }^{13}$, métaphores et analogies sont identiquement mobilisées dans les régimes d'argumentation de l'individu lambda $a^{14}$ et dans ceux qui promeuvent la création artistique et scientifique ${ }^{15}$.

11 C'est pourquoi la distinction analytique entre représentations (en tant que croyances qui peuvent se révéler fausses) et connaissances (en tant que croyances vraies justifiées de manière fiable) n'a guère de pertinence empirique. Nous parlerons de représentations plutôt que de connaissances, car l'ensemble des secondes est contenu dans l'ensemble des premières, et parce que ce qui nous intéresse ici n'est pas le problème poppérien de la « démarcation ", mais le fait que les représentations sont agissantes en ce qu'elles déterminent les comportements des acteurs indépendamment de leur éventuelle teneur en vérité. En d'autres termes, notre perspective est ici pragmatique, et non normative.

12 Denis Zwirn et Hervé Zwirn, "La révision des croyances ", Pour la science, $n^{\circ} 311$, septembre, 2003, p. 64-69.

13 George Lakoff et Mark Johnson, Metaphors We Live By, Chicago, The University of Chicago, 1980; George Lakoff et Mark Johnson, Philosophy in the Flesh. The Embodied Mind and Its Challenge to Western Thought, New York, Basic Books, 1999; Douglas Hofstadter et Emmanuel Sander, L'analogie, cour de la pensée, Paris, Odile Jacob, 2013.

14 Andrew Ortony (dir.), Metaphor and Thought, Cambridge, Cambridge University Press, 1993; Marie-Dominique Gineste, Analogie et cognition. Étude expérimentale et simulation informatique, Paris, Presses Universitaires de France, 1997.

15 Cf. Arthur I. Miller, op. cit. Qu'il s'agisse de nos plaidoyers les plus quotidiens ou d'argumentations déployées dans le cadre des sciences de la nature ou dans celui des sciences humaines et sociales, analogies et métaphores sont utilisées partout afin d'emporter la conviction. Cf. Fernand Hallyn, Les structures rhétoriques de la science. De Képler à Maxwell, Paris, Seuil, 2004; André Lichnerowicz, François Perroux et Gilbert Gadoffre (dir.), Analogie et 
Enfin, une troisième raison de mettre sur le même pied acteurs académiques et profanes consiste en ce que les capacités d'apprentissage sont distribuées de la même manière parmi tous les acteurs. Tous font preuve des mêmes capacités hiérarchisées en une pluralité théorique de niveaux logiques, dont trois sont particulièrement significatifs d'un point de vue empirique ${ }^{16}$ : celui qui consiste en l'acculturation individuelle ou collective d'une vision donnée du monde (apprentissage de niveau 2 ou secondaire), consécutive à la réception itérée d'un certain type d'informations (apprentissage de niveau 1 ou primaire) que cette vision du monde vient en retour contextualiser; celui qui consiste en une mutation brutale de cette vision du monde (apprentissage de niveau 3), qui installe un nouveau contexte épistémique accueillant de nouveaux apprentissages primaires et secondaires ${ }^{17}$. En réalité, ces processus d'apprentissage sont communs à tous les acteurs considérés comme autant de systèmes adaptatifs complexes au sens de Murray Gell-Mann ${ }^{18}$. De tels systèmes obtiennent de l'information à propos de leur environnement et de leurs interactions avec celui-ci (apprentissage primaire), identifient des régularités au sein de cette information et condensent ces régularités en formulant des modèles afin d'agir dans le monde réel sur la base de ces derniers (apprentissage secondaire). Dans chaque cas, il existe plusieurs modèles en compétition, et l'action dans le monde réel exerce une influence rétroactive sur cette compétition. Plus précisément, chacun de ces modèles s'enrichit alors d'informations supplémentaires,

connaissance, Tome I - Aspects historiques, Paris, Éditions Maloine, 1980; Marie-José Durand-Richard et al., L'analogie dans la démarche scientifique. Perspective historique, Paris, L'Harmattan, 2008; Michel de Coster, L'analogie en sciences humaines, Paris, Presses Universitaires de France, 1978.

16 Gregory Bateson, Steps to an Ecology of Mind, New York, Chandler Publishing Company, 1972.

17 C'est sur la base de cette catégorisation qu'Erving Goffman a introduit la frame analysis en sciences sociales : ce qu'il nomme les "cadres primaires » et les « ruptures de cadres » désigne respectivement les apprentissages batesoniens de niveaux 2 et 3. Cf. Erving Goffman, Frame Analysis: An Essay on the Organization of Experience, New York, Harper \& Row, 1974.

18 Murray Gell-Mann, Le quark et le jaguar. Voyage au cœur du simple et du complexe, Paris, Albin Michel, 1995 [1994]. 
parmi lesquelles figurent celles qui avaient été négligées lors de l'extraction de régularités à partir du flux de données initialement observables (possibilité d'apprentissage de niveau 3). Et ceci afin d'obtenir un résultat applicable au " monde réel ", c'est-à-dire la description d'un système observé, la prédiction d'événements, ou l'indication d'un comportement pour le système adaptatif complexe lui-même. Cette description très générale vaut pour les processus d'apprentissage de tous les acteurs, notamment la fraction de ces derniers qui est spécialisée dans ce que Murray Gell-Mann appelle "l'entreprise scientifique". Les modèles sont ici des théories, et ce qui arrive dans le "monde réel » est la confrontation entre théories et observations. De nouvelles théories peuvent venir concurrencer celles qui existent déjà, engageant ainsi une compétition basée sur la cohérence et le degré de généralité de chacune, et dont le résultat dépendra finalement de leurs capacités respectives d'expliquer les observations existantes et de prédire correctement de nouvelles observations. Chaque théorie de cette sorte constitue une description hautement condensée d'une classe très nombreuse de situations, et doit donc être complétée par la description détaillée de chaque situation particulière pour pouvoir donner lieu à des prédictions spécifiques $^{19}$.

Tous les acteurs individuels peuplant nos sociétés seront donc considérés ici comme de tels systèmes, et si nous distinguerons à l'occasion les acteurs académiques au sein de cette population globale, ce sera uniquement afin de mettre en exergue certaines évolutions qui concernent l'ensemble de cette dernière, mais se laissent mieux entrevoir dans ce sous-ensemble d'acteurs provisoirement isolé. Dans le champ ainsi défini, nous utiliserons une modélisation de la structure et de l'évolution d'un réseau sociocognitif complexe d'acteurs hétérogènes parmi lesquels le savoir est distribué et situé ${ }^{20}$, et poursuivrons une réflexion engagée plus

19 Murray Gell-Mann, op. cit., p. 33 sq., p. 94.

20 Bernard Ancori, "Espace-temps d'un réseau sociocognitif complexe. I. Propension à communiquer et présent spécieux ", Nouvelles perspectives en sciences sociales. Revue internationale de systémique complexe et d'études relationnelles, vol. 3, n 2, 2008, p. 113-181; Bernard Ancori, "Espace-temps d'un 
récemment sur la production et la circulation des savoirs dans nos sociétés ${ }^{21}$.

Dans une première section, nous traiterons de deux dilemmes interdépendants. Le premier concerne le couple " créativitél proximité ", et il est lié à la distribution des représentations parmi les acteurs individuels au sein du réseau : plus cette distribution est celle d'acteurs cognitivement proches, plus elle favorise la communication parmi ces derniers, mais moins cette communication sera créative - et inversement. Le second dilemme concerne le couple " communication/cognition ", et il caractérise le choix auquel se trouve confronté l'acteur individuel dans chaque état donné du réseau : communiquer, et produire ainsi de la nouveauté en un sens faible au sein d'un réseau qui n'évolue alors qu'en actualisant des représentations qui y sont déjà virtuellement présentes, ou cogiter, et produire ainsi de la nouveauté en un sens fort en venant abonder le volume des représentations virtuelles et actuelles du réseau.

Dans une seconde section, nous attribuerons un essoufflement récent de la créativité dans nos sociétés techniciennes, révélé par l'histoire des sciences comme par l'analyse bibliométrique, au privilège croissant que ces sociétés conferent depuis peu à la communication par rapport à la cognition. In fine, nous serons ainsi conduit à plaider en faveur de l'instauration de collaborations systématiques et efficaces entre le monde académique et les mondes profanes, car de telles collaborations constituent le gage d'une créativité individuelle et collective revivifiée au sein de notre réseau global d'acteurs individuels.

réseau sociocognitif complexe. II. Temporalités historiques et entropie sociocognitive ", op. cit.

Bernard Ancori, "Expertise et citoyenneté : les Grecs anciens et nous. De l'Agora antique aux forums hybrides modernes ", Revue d'Anthropologie des Connaissances, vol. 3, $\mathrm{n}^{\circ}$ 3, Paris, 2009, p. 485-529; Bernard Ancori, " La production et la circulation des connaissances scientifiques et des savoirs profanes dans nos sociétés techniciennes ", dans François Darbellay (dir.), La circulation des savoirs. Interdisciplinarité, concepts nomades, analogies, métaphores, Berne, Éditions scientifiques internationales Peter Lang, 2012, p. 203-240. 


\section{Sur deux dilemmes concernant la créativité}

Le dilemme " créativité/proximité "

Existe-t-il une structure optimale de notre réseau en terme de productivité de la communication entre acteurs, source de nouvelles représentations individuelles et collectives? Cette question nous confronte immédiatement au dilemme " créativité/proximité » qui ouvre cette section. En effet, s'il est vrai que la production potentielle de représentations nouvelles liée à la communication entre acteurs est d'autant plus importante que les univers cognitifs de ces derniers sont a priori différents, d'un autre côté, plus ces univers sont différents, moins l'établissement de communications entre les acteurs concernés est aisé : la pertinence empirique du fameux proverbe "qui se ressemble s'assemble " est largement confirmée par les données sociologiques qui montrent les relations affinitaires présentées pour principale caractéristique d'être homophiles ${ }^{22}$. De sorte que la structure optimale recherchée devrait être intermédiaire entre celles de deux ensembles d'états du réseau : ceux où l'hétérogénéité des univers cognitifs des acteurs est telle que leurs communications, potentiellement très productrices de représentations nouvelles, sont relativement rares ou improbables (voire impossibles) du fait des dissemblances de ces univers, et ceux où les univers cognitifs des acteurs sont au contraire si proches (à la limite identiques) que leurs communications relativement fréquentes ou probables (à la limite certaines) sont d'autant plus pauvres en représentations nouvelles que les ressemblances entre acteurs sont affirmées (à la limite parfaites) ${ }^{23}$.

22 Cf. Alain Degenne et Michel Forsé, « Comment on trouve ses amis. Enquête sur la sociabilité des Français ", dans Les liens sociaux invisibles, numéro hors série de Sciences Humaines, n 5, mai-juin 1994, p. 20-24; Alain Degenne et Michel Forsé, Les Réseaux sociaux, Paris, Armand Colin, 1994.

23 Chaque état du réseau est représenté par une matrice $(\mathrm{m}, \mathrm{n})$ dont les lignes sont les acteurs $A_{i}: \mathrm{i}=1, \ldots, \mathrm{m}$, et les colonnes des catégories psychologiques $C_{j}: \mathrm{j}=1, \ldots, \mathrm{n}$. Les coefficients $\left[\mathrm{a}_{\mathrm{ij}}\right]$ de cette matrice prennent la valeur 1 lorsque $A_{i}$ connaît $C_{j}$ et 0 dans le cas inverse. 
Nous retrouvons ainsi une idée formulée par Claude LéviStrauss lors de son quatrième séjour au Japon, au printemps 1986, où il s'interrogeait sur le risque de perte de diversité culturelle engendrée par la rapidité accrue des moyens de transport et de communication, synonyme d'homogénéisation croissante des cultures alignées sur le modèle créé par les bouleversements de la civilisation industrielle en Occident. Il écrivait alors :

Les grandes époques créatrices furent celles où la communication était devenue suffisante pour que des partenaires éloignés se stimulent, sans être cependant assez fréquente et rapide pour que les obstacles, indispensables entre les individus comme entre les groupes, s'amenuisent au point que des échanges trop faciles uniformisent et confondent cette diversité ${ }^{24}$.

Or, nous avons montré ailleurs ${ }^{25}$ que l'évolution la plus probable d'un réseau sociocognitif d'acteurs individuels dont le seul moteur serait la communication est telle qu'à partir d'un état initial où les proximités cognitives entre acteurs sont distribuées de manière homogène parmi ces derniers, le réseau tend spontanément vers une constellation d'amas d'acteurs ${ }^{26}$. Au sein de cette constellation, les acteurs d'un amas donné sont cognitivement de plus en plus proches (et communiquent donc de plus en plus facilement entre eux) et de plus en plus distants des acteurs des autres amas (avec lesquels ils communiquent donc de plus en plus difficilement). Le nombre d'acteurs du réseau ainsi que leurs représentations initiales étant supposés donnés ${ }^{27}$, tant que n'a pas

24 Claude Lévi-Strauss, L'anthropologie face aux problèmes du monde moderne, Paris, Seuil, 2011, p. 142.

25 Bernard Ancori, "Espace-temps d'un réseau sociocognitif complexe. I. Propension à communiquer et présent spécieux ", op. cit.

26 "Homogène » signifie ici que les représentations des acteurs sont alors telles que tous disposent d'une égale quantité d'information, et que chaque acteur est à égale proximité cognitive de tous les autres.

27 Les représentations des acteurs sont composées de catégories mentales, c'est-à-dire de significations de concepts mentaux vus sous l'angle de leur extension (l'extension du concept "fleur " est l'ensemble de toutes les fleurs). Plus précisément, ce sont les représentations occurrences de fleurs particulières, et non ces fleurs elles-mêmes, qui « tombent sous » la catégorie "fleur ». La notion de catégorie mentale s'applique donc aussi bien à des 
émergé un langage partagé par tous les acteurs sous forme de représentations simultanément présentes dans l'ensemble de leurs univers cognitifs, la productivité de la communication en termes de représentations nouvelles décroît constamment tout au long de ce processus qui se révèle cumulatif. Et lorsqu'a émergé un langage partagé entre tous les acteurs, de sorte que chacun de ces derniers peut à présent s'évader de son amas pour communiquer avec les acteurs de tous les autres amas, le réseau tend extrêmement rapidement vers un état final consistant en un seul et même amas d'acteurs strictement identiques ${ }^{28}$. Le réseau a atteint ainsi

représentations de nature perceptive qu'à des représentations proprement conceptuelles. Par ailleurs, une occurrence est une réalité physique perçue qui est cause d'une suite d'événements mentaux, perceptifs puis sémantiques, dont le produit global est une "représentation occurrence ", et la catégorisation est définie comme la mise en œuvre de la relation " est-un " (" ceci est une fleur "). La catégorisation consiste donc à affecter une représentation occurrence particulière (" cet objet, actuellement en face de moi...») à une représentation générique, qui est la catégorie (..." est une fleur "). Cf. Jean-François Le Ny, Comment l'esprit produit du sens. Notions et résultats des sciences cognitives, Paris, Odile Jacob, 2005, p. 160 sq. Dans cette conception, la perception est entièrement tissée de catégorisations, au sens où les acteurs perçoivent des objets (choses, individus, événements, etc.) regroupés dans des catégories perceptives : percevoir est reconnaître une occurrence relevant d'une catégorie (ibid., p. 164 sq.).

Soit un réseau dont l'état initial est homogène au sens donné ici à ce terme, et où chacun des $m$ acteurs connaît le même nombre minimal de catégorie mentales - état initial oblige; le nombre total de catégories présentes dans la "mémoire " globale du réseau est alors égal à $n=3 \mathrm{~m}$. Une série de simulations informatiques a été lancée sur la base d'un algorithme correspondant à un tel état, avec des contenus des communication dyadiques tels que chaque message émis contenait deux catégories exactement, dont l'une était simultanément déjà connue des deux protagonistes (ce qui a rendu leur communication possible) et l'autre était inconnue du récepteur de chaque message (ce qui a permis à chacun d'apprendre). Cette série de simulations a montré que de telles communications aboutissaient en un peu moins de $3 m$ périodes à l'établissement d'un langage partagé par tous les acteurs, et en un peu plus de $3 m$ périodes à un état final tel que les représentations de tous les acteurs étaient strictement identiques. En d'autres termes, dans ces conditions il s'écoule très peu de temps entre le moment où les acteurs disposent d'un langage partagé par tous $\left(\exists \mathrm{C}_{\mathrm{j}}\right.$ telle que $\left[\mathrm{a}_{\mathrm{ij}}\right]=$ $1, \forall \mathrm{i}$ ), et celui où tous les acteurs ont strictement les mêmes représentations $\left(\left[\mathrm{a}_{\mathrm{ij}}\right]=1, \forall \mathrm{i}, \forall \mathrm{j}\right)$. Cf. Bruno Belarte, Simulation de comportements sociaux dans un réseau d'agents individuels, Mémoire de TER, Fouille de Données et Bioinformatique Théorique du Laboratoire des Sciences de l'Image, de 
un équilibre informationnel final, au sens où la productivité de la communication en son sein est devenue strictement et irrévocablement nulle : aucune représentation nouvelle ne peut plus y apparaître. C'est très précisément ce que craignait Claude LéviStrauss lorsqu'il écrivait :

Le jeu en commun, d'où résulte tout progrès, doit entraîner, à échéance plus ou moins brève, une homogénéisation des ressources de chaque joueur. Si la diversité est une condition initiale, il faut reconnaitre que les chances de gain deviennent d'autant plus faibles que la partie doit se prolonger $^{29}$.

La raison profonde d'une telle évolution tient au fait que la communication entre acteurs n'est créative qu'en un sens faible, car les représentations nouvelles qu'elle produit ne sont jamais que des actualisations de combinaisons de catégories mentales déjà présentes à l'état virtuel au sein du réseau considéré globalement. Montrons-le en précisant la notion de mémoire individuelle qui fonctionne dans notre modèle. Propre à chaque acteur, cette mémoire ne se réduit pas à l'ensemble des catégories mentales connues de lui, mais consiste plutôt, dans sa plus grande extension, en l'ensemble des parties de cet ensemble ${ }^{30}$. Ainsi, si l'acteur $A_{i}$ connaît à la date $\mathrm{t}_{0}$ les catégories $\mathrm{C}_{1}, \mathrm{C}_{3}$ et $\mathrm{C}_{5}$, son lexique est représenté par l'ensemble des parties de l'ensemble $\left\{\mathrm{C}_{1}, \mathrm{C}_{3}, \mathrm{C}_{5}\right\}$, et à supposer que toutes ces parties soient sémantiquement pertinentes, il en va de même pour sa mémoire : $\left\{\varnothing, \mathrm{C}_{1}, \mathrm{C}_{3}, \mathrm{C}_{5}, \mathrm{C}_{1} \mathrm{C}_{3}, \mathrm{C}_{1} \mathrm{C}_{5}, \mathrm{C}_{3} \mathrm{C}_{5}, \mathrm{C}_{1} \mathrm{C}_{3} \mathrm{C}_{5}\right\}$

Il en va de même pour un acteur $A_{k}$ qui connaît à la même date les catégories $\mathrm{C}_{2}, \mathrm{C}_{3}$ et $\mathrm{C}_{4}$. Son lexique et sa mémoire sont représentés par :

l'Informatique et de la Télédétection (FDBT-LSIIT, UMR 7002 CNRSUniversité de Strasbourg, avril 2010.

29 Claude Lévi-Strauss, op. cit., p. 142.

30 Nous disons "dans sa plus grande extension ", car toutes ces parties formellement constructibles n'ont pas nécessairement de pertinence sémantique pour l'acteur considéré dans l'état actuel du réseau. Il nous faut donc distinguer les lexiques accessibles aux acteurs considérés (ces ensembles de parties) des mémoires individuelles de ces acteurs (les sous-ensembles sémantiquement pertinents de ces lexiques). Néanmoins, cette distinction n'étant pas pertinente pour notre présent propos, nous n'en tiendrons pas compte ici. 
$\left\{\varnothing, \mathrm{C}_{2}, \mathrm{C}_{3}, \mathrm{C}_{4}, \mathrm{C}_{2} \mathrm{C}_{3}, \mathrm{C}_{2} \mathrm{C}_{4}, \mathrm{C}_{3} \mathrm{C}_{4}, \mathrm{C}_{2} \mathrm{C}_{3} \mathrm{C}_{4}\right\}$

Quant à la "mémoire " du réseau global, elle consiste ici en l'union de ces deux mémoires individuelles. Il est facile de voir qu' elle comporte 14 parties à la date $\mathrm{t}_{0}$. Or, le nombre de parties de l'ensemble de toutes les catégories mentales présentes à cette date dans la mémoire d'au moins un acteur - l'ensemble des parties de l'ensemble $\left\{\mathrm{C}_{1}, \mathrm{C}_{2}, \mathrm{C}_{3}, \mathrm{C}_{4}, \mathrm{C}_{5}\right\}-$ est, lui, égal à 32. À la date $\mathrm{t}_{0}$, il existe donc à l'état virtuel 18 combinaisons de catégories mentales dans le réseau, c'est-à-dire un "réservoir » de combinaisons qui y sont déjà présentes, mais non encore actualisées. La communication entre acteurs a pour seul effet d'actualiser ces combinaisons de catégories, et c'est pourquoi elle n'est créative qu'en un sens faible : elle ne produit de l'information qu'en épuisant progressivement un réservoir de représentations qui existe d'emblée dans le réseau global. Supposons ainsi qu'entre $\mathrm{t}_{0}$ et $\mathrm{t}_{1}$, la communication établie entre les acteurs $A_{i}$ et $A_{k}$ soit telle que $A_{i}$ communique le message " $\mathrm{C}_{1} \mathrm{C}_{3}$ " à $A_{k}$ qui lui communique simultanément les message « $\mathrm{C}_{2} \mathrm{C}_{3}$ ". À la date $\mathrm{t}_{1}$, la mémoire individuelle de chaque acteur contient 16 combinaisons de ce type, et la " mémoire " du réseau global en contient 24 : de $\mathrm{t}_{0}$ à $\mathrm{t}_{1}$, la communication a actualisé 10 combinaisons de catégories mentales, de sorte qu'à la date $t_{1}$ le réservoir précité n'en contient plus que 8 - et plus aucune à la date $\mathrm{t}_{2}$ pour peu qu'entre $\mathrm{t}_{1}$ et $\mathrm{t}_{2}, A_{i}$ communique le message " $\mathrm{C}_{3} \mathrm{C}_{5}$ " à $A_{k}$ qui lui communique simultanément le message $" \mathrm{C}_{3} \mathrm{C}_{4}$ ".

En résumé, si la communication entre les acteurs individuels était le seul moteur de créativité dans nos sociétés, la trajectoire la plus probable de ces dernières les mènerait inéluctablement vers une homogénéisation totale des univers cognitifs de l'ensemble des acteurs individuels qui les composent (lorsqu'aurait émergé un langage partagé par tous ces derniers), en passant par une tendance vers une homogénéisation croissante des univers cognitifs des acteurs contenus dans chaque amas de la constellation d'univers cognitifs peuplant ces sociétés (avant l'émergence d'un langage partagé par tous les acteurs). Surviendrait nécessairement, à plus ou moins long terme, un état dans lequel la 
productivité de la communication en représentations nouvelles serait devenue strictement nulle. Le défi impliqué par le dilemme " créativité/proximité » est donc celui d'assurer en permanence un réglage fin de la structure du réseau entre univers cognitifs suffisamment différents pour assurer une productivité optimale en termes de représentations nouvelles à la faveur des communications établies entre acteurs, mais aussi suffisamment proches pour que la structure des probabilités de communications entre acteurs permette à ces communications de s'établir facilement. Nous y reviendrons.

Le dilemme « communication/cognition»

Afin d'éviter au réseau la survenue d'un équilibre informationnel final analogue à la mort entropique d'un système thermodynamique, il faut introduire dans son évolution un autre moteur de créativité (au moins) à côté de celui que constituerait la seule communication entre acteurs dont la population et les mémoires individuelles seraient données. Il existe pour cela deux possibilités. La première consisterait à introduire des générations d'acteurs individuels dans le réseau, au lieu de considérer leur population comme donnée. Avec l'arrivée de chaque génération nouvelle, comme avec le départ de chaque génération ancienne, certaines modifications affecteraient mécaniquement l'ensemble des catégories mentales figurant dans la "mémoire " globale du réseau - certaines d'entre-elles disparaissant, d'autres faisant leur apparition - et renouvelleraient ipso facto le " réservoir " de combinaisons virtuelles non encore actualisées. La seconde possibilité, que nous privilégierons ici, consiste à introduire la production de catégories nouvelles via la formulation d'analogies ou de métaphores inédites. Cette catégorisation présente deux faces indissolublement liées. L'une est celle de raffinements de catégories existantes, telle celle qui voit l'acteur $A_{i}$ distinguer à présent les " roses " des «tulipes " au sein des "fleurs » qu'il considérait précédemment tout uniment. Il se crée alors deux colonnes supplémentaires dans la matrice représentant l'état correspondant du réseau, et si l'acteur $A_{i}$ est seul à avoir opéré ce raffinement, 
ces deux colonnes ne comportent chacune qu'un seul «1", situé à leurs croisements avec la $i$-ème ligne, leurs croisements avec les $m-1$ autres lignes étant peuplés de « 0 » - ce qui diffère d'autant la survenue de la mort entropique du réseau. L'autre face de la catégorisation est celle de la production de nouvelles catégories par agrégation de catégories existantes, telle la catégorie "végétaux " obtenue par agrégation des catégories "fleurs " et " légumes ", et correspondant à l'apparition d'une colonne supplémentaire dans le réseau, avec le même type de distribution de « 1 » et de « 0 » que dans le cas du raffinement. La complémentarité de ces deux gestes cognitifs - raffinement et agrégation - est absolue. En effet, au cœur même du raffinement, c'est encore une forme d'agrégation qui opère : distinguer à présent les " roses " des " tulipes " là où l'on ne voyait auparavant que des "fleurs " implique de discerner certaines analogies entre les représentations occurrences de la catégorie " roses » et d'agréger celles-là au sein de celle-ci, et il en va de même des analogies différentes que présentent symétriquement l'ensemble des représentations occurrences de la catégorie "tulipe ». C'est bien sur la base de leurs caractéristiques analogues que la pensée agrège en chacune de ces catégories leurs représentations occurrences respectives. Mais, d'un autre côté, le raffinement opère également au cour de l'agrégation qui avait produit la catégorie "fleur " elle-même, au sens où celle-ci est issue par exemple de sa distinction d'avec la catégorie "légume " au sein de la catégorie "végétaux ", elle-même distinguée de la catégorie " animaux " au sein de la catégorie "vivants ", elle même distinguée de la catégorie " inanimés ", etc. En d'autres termes, comme le montrent à loisir Douglas Hofstadter et Emmanuel Sander, le moteur de la pensée consiste en l'analogie qui ne cesse de catégoriser en opérant des agrégations ininterrompues qui vont de pair avec des raffinements tout aussi ininterrompus - " un peu comme si un immeuble transformait les briques avec lesquelles il est construit ${ }^{31}$ ».

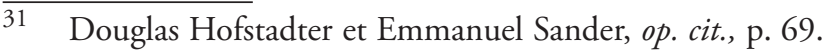


Succession de générations d'acteurs et production d'analogies créatrices de nouvelles catégories mentales : il est clair que tout réseau concret d'acteurs individuels connaît simultanément ces deux modalités. Leur effet y est de diversifier en permanence la distribution des représentations au sein du réseau, donc d'y contrecarrer ipso facto l'effet homogénéisant de la communication sociale. S'agissant d'un réseau comportant une population donnée de ces derniers, nous sommes ainsi confrontés à un second dilemme dans la problématique générale de la créativité : le dilemme " communication/cognition ". Il ne s'agit évidemment pas d'opposer ici communication et cognition au sens où ces deux activités seraient mutuellement exclusives : communiquer signifie toujours aussi cogiter - ne serait-ce que sous forme de rappel ou d'inscription des catégories véhiculées par les messages émis ou reçus dans les mémoires des protagonistes de la communication - et, réciproquement, cogiter signifie toujours aussi communiquer avec soi-même. Néanmoins, chaque réalisation concrète de l'une ou l'autre de ces deux activités modifie l'état actuel du réseau, et, ce faisant, fait passer ce dernier d'une date donnée à la date suivante. Il s'ensuit que, dans chaque état donné du réseau, les acteurs effectuent l'une ou l'autre.

Contrairement à la communication, la cognition productive d'analogies ou de métaphores inédites est créative en un sens fort, puisqu'elle débouche sur l'apparition de nouvelles catégories mentales dans le réseau, et par suite sur celle de nouvelles combinaisons (virtuelles ou actualisées) de telles catégories dans la "mémoire " globale de ce dernier ${ }^{32}$. À travers les apprentissages qu'il permet de réaliser, ce type de cognition alimente sans cesse nos sociétés en représentations inédites qui viennent renouveler les contenus des messages échangeables lors des communications entre acteurs. Accroissant la diversité au sein de la distribution globale des représentations, il contrebalance constamment l'effet homogénéisant que la communication exerce sur cette distribution. Il s'ensuit que si les acteurs communiquent plus volontiers

$\overline{32}$ Bernard Ancori, "Analogie, évolution scientifique et réseaux complexes ", op. cit. 
qu'ils ne cogitent en produisant de nouvelles catégories mentales, ils impriment au réseau une évolution qui le mène vers la mort entropique évoquée ci-dessus, alors que s'ils agissent à l'inverse, ils contrecarrent cette évolution en différant la survenue d'un tel état final. Le réglage fin de la structure du réseau assurant une productivité optimale de ce dernier tout au long de sa trajectoire dépend donc de manière critique de la comparaison de deux variables : le taux de socialisation de catégories mentales existantes via la communication, et le taux de création de nouvelles catégories mentales via la cognition productrice d'analogies et de métaphores inédites. Plus précisément, dans chaque état du réseau, ces taux doivent être tels qu'ils permettent à ce dernier de conserver tout au long de sa trajectoire un état analogue à son état initial caractérisé par une distribution homogène des proximités/distances cognitives et des catégories entre tous les acteurs.

Le choix de l'acteur individuel entre ces deux activités possibles s'effectue dans chaque état du réseau, mais il n'est pas indépendant de sa situation propre au regard de la distribution des propensions à communiquer dans l'état considéré. Considérons ainsi un réseau qui comporterait un nombre impair d'acteurs, et dont l'état initial serait caractérisé par une distribution homogène des propensions à communiquer entre ces derniers. Etant donné que l'évolution la plus probable du réseau résulte de communications dyadiques ${ }^{33}$, un acteur au moins se trouverait alors écarté de toute communication. Cet isolement peut n'être que momentané, étant donné que toute communication établie par au moins un autre acteur au sein des $(m-1) / 2$ couples en communication suffit à modifier l'ensemble de la distribution des propensions à communiquer au sein du réseau - y compris celles de l'acteur momentanément écarté de toute communication. Dans le nouvel

33 En effet, la propension à communiquer entre trois acteurs quelconques $A_{k}$, $A_{l}$ et $A_{m}$ est égale au produit des propensions à communiquer de ces trois acteurs pris deux à deux : $\mathrm{p}_{\mathrm{kl}} \cdot \mathrm{p}_{\mathrm{lm}} \cdot \mathrm{p}_{\mathrm{mk}}$. Or, tous les termes de ce produit sont inférieurs à l'unité, de sorte qu'il est toujours inférieur à chacun de ses termes. Cela ne signifie évidemment pas qu'il n'existerait que des communications dyadiques dans chaque état du réseau, mais seulement que ces dernières sont toujours plus fréquentes que des communications établies entre acteurs en nombres plus élevés. 
état du réseau ainsi engendré, la distribution des propensions à communiquer peut alors être devenue plus favorable à l'établissement d'une communication par l'acteur précédemment isolé. De fait, le modèle montre que tel serait effectivement le cas si l'acteur en question s'abstenait de toute activité durant la période considérée : la nouvelle distribution des représentations serait alors telle que ses propensions à communiquer avec les acteurs de chacun des deux amas les plus proches de lui seraient devenues supérieures aux propensions à communiquer existant désormais entre les acteurs appartenant à ces deux amas différents ${ }^{34}$. Mais il est difficile d'imaginer qu'un acteur puisse s'abstenir de toute activité sous prétexte qu'il serait momentanément exclu de toute communication! Il est davantage vraisemblable qu'il profiterait de son isolement provisoire pour cogiter, et éventuellement créer ainsi de nouvelles catégories mentales - qui seraient dans ce cas idiosyncrasiques. Et il se peut dès lors que cette activité cognitive débouche sur la création d'un nombre de catégories idiosyncrasiques suffisant pour venir diminuer encore chacune des propensions à communiquer de l'acteur considéré avec tous les autres. C'est alors un processus de marginalisation de l'acteur considéré qui s'amorcerait au sein du réseau.

Restreignons pour l'instant notre réseau au seul monde académique. Le processus de marginalisation évoqué à l'instant est potentiellement cumulatif, et il peut ainsi conduire à la forme d'auto-exclusion qui alimente les bataillons d'une "alterscience " dont les représentants bâtissent de véritables constructions théoriques à l'appui de leur croyance ou de leur idéologie, constructions dont le rejet ou la simple absence de reconnaissance de la part de leur communauté scientifique d'origine les amène à invoquer une théorie du complot visant leurs idées ${ }^{35}$. En fait, nous sommes ici en présence d'acteurs dont l'isolement initial par rapport à leurs communautés scientifiques respectives ne s'est pas simultanément accompagné d'un rapprochement avec

34 Bernard Ancori, "Espace-temps d'un réseau sociocognitif complexe. I. Propension à communiquer et présent spécieux ", op. cit.

35 Cf. Alexandre Moatti, Alterscience. Postures, dogmes, idéologies, Paris, Odile Jacob, 2013. 
d'autres communautés scientifiques, avec les effets d'ouverture et de changement de leurs cadres cognitifs qu'un tel rapprochement aurait pu entraîner. De ce fait, aucune rupture de cadre ou apprentissage de niveau 3 n'a pu s'effectuer, et les acteurs concernés sont ainsi restés prisonniers de leurs cadres primaires, voire pris dans un processus d'apprentissage secondaire poussé jusqu'à la limite où il aboutit à ancrer une routine cognitive dans les profondeurs non conscientes de leur esprit - une routine rendue ainsi très difficile, voire impossible, à extirper. À l'inverse, l'amorce du même type de marginalisation peut déboucher sur une extension du champ de ce qui est considéré comme "scientifique ", et l'on peut alors parler de "marginalité créatrice ${ }^{36}$ ". Tel fut le cas d'un Claude Lévi-Strauss profitant de son exil momentané à New York au début de la Seconde Guerre mondiale pour renouveler la réflexion anthropologique grâce à son usage de la linguistique de Jakobson et de Saussure, sa proximité avec le surréalisme et la familiarité qu'il a alors acquise avec la tradition anthropologique américaine ${ }^{37}$. Entre ces deux cas extrêmes, nous pourrions parler de " marginalité faiblement créatrice " à propos de cet autre exilé new-yorkais à la même époque, Georges Gurvitch, qui n'exploita guère cette situation pour sortir de son secteur professionnel d'origine, de sorte que le cours de sa trajectoire sociale et scientifique resta inchangé - sa trajectoire ellemême restant néanmoins scientifique ${ }^{38}$.

À travers ces trois cas de figure, il apparaît clairement que nos deux dilemmes " créativité/proximité » et " communication/ cognition " sont largement interdépendants, et que la forme de cette interdépendance trouve une traduction immédiate en termes de créativité. Ainsi, les représentants de l'« alterscience " ont durablement privilégié la cognition par rapport à la

\footnotetext{
36 Mattei Dogan et Robert Pahre, Linnovation dans les sciences sociales. La marginalité créatrice, Paris, Presses Universitaires de France, 1991.

37 Geoffroy de Lagasnerie, op. cit., p. 102-105.

38 Laurent Jeanpierre, "Une opposition structurante pour l'anthropologie structurale : Lévi-Strauss contre Gurvitch, la guerre de deux exilés français aux États-Unis ", Revue d'histoire des sciences humaines, $\mathrm{n}^{\circ}$ 11, 2004, p. $13-43$.
} 
communication, ce qui les a progressivement éloignés des autres acteurs de leur amas initial au sein du réseau; or, cet éloignement ne s'est pas accompagné d'un rapprochement de leur part vers d'autres acteurs composant d'autres amas, et le privilège ainsi donné à la cognition sur la communication s'est accentué sur un mode cumulatif qui a contraint leurs processus cognitifs à fonctionner de plus en plus en circuit fermé, ce qui en retour les a toujours davantage privés de communications qui leur auraient permis de réviser leurs " cadres primaires ", etc., de sorte qu'ils ont fini par fonctionner sur un mode sectaire. À l'inverse, la marginalisation provisoire de Claude Lévi-Strauss par rapport aux réseaux de l'anthropologie française d'avant la Seconde Guerre mondiale l'a conduit à communiquer avec d'autres réseaux, et la rupture de cadre ainsi provoquée l'a finalement conduit à formuler des catégorisations nouvelles sur la base d'analogies et de métaphores inédites qui ont profondément renouvelé la réflexion anthropologique. Enfin, placé dans la même situation d'exil que Claude Lévi-Strauss, Georges Gurvitch est pour sa part resté pris dans son secteur professionnel d'origine, où il se borna «à reprendre et approfondir [...] des problématiques et des thématiques anciennes, sans hybrider sa pensée avec la tradition sociologique américaine et sans innover vraiment ${ }^{39}$ ».

Trois leçons peuvent être tirées de ces exemples : $i$ ) le fait de beaucoup cogiter et de peu communiquer peut conduire l'acteur à s'enfermer dans un amas constitué à la limite de lui seul - et toute créativité disparaît alors de son activité : la créativité forte liée à la cognition est annulée par l'absence de la créativité faible associée à la communication; ii) le fait d'établir des communications avec de multiples acteurs provenant d'autres amas que son

$39 \quad$ Laurent Jeanpierre, $o p$. cit., p. 24. Nous remercions le rapporteur anonyme qui a tenu à nuancer ce jugement en indiquant qu'à son retour des ÉtatsUnis, Georges Gurvitch créa, sous l'influence de la sociologie américaine, le Centre d'Études Sociologiques au CNRS, qui fut l'incubateur de ce qui allait devenir la sociologie d'enquête moderne. Néanmoins, cette remarque n'affecte que marginalement notre propos : s'il l'a ainsi enrichie, Georges Gurvitch resta cantonné à sa discipline, alors que le bouleversement de l'anthropologie par Claude Lévi-Strauss fut le fruit de l'interdisciplinarité pratiquée par ce dernier. 
amas initial conduit l'acteur à construire des catégorisations inédites, et cette créativité forte se manifeste par un changement de paradigme : plus les amas avec lesquels l'acteur entre en contact sont divers, plus la créativité faible de la communication favorise la créativité forte de la cognition; iii) le fait de n'établir de communication que dans le cadre restreint du même amas d'acteurs se traduit chez l'acteur par une activité cognitive dont la créativité reste faible, car elle se borne à apporter des perfectionnements mineurs à un savoir déjà établi dans le cadre d'un paradigme donné : la créativité toujours plus faible de la communication inhibe toujours davantage la créativité forte de la cognition. En somme, communiquer sans cogiter conduit à la mort du réseau sous forme de disparition irrévocable de toute créativité individuelle ou collective; cogiter sans communiquer mène à cette forme de mort individuelle que représente la disparition de toute créativité chez l'acteur concerné; communiquer et cogiter débouche sur une créativité d'autant plus forte que les communications de l'acteur concerné s'établissent avec des acteurs d'amas suffisamment divers. Qu'en est-il de l'interdépendance entre ces deux dilemmes dans le cadre de nos sociétés techniciennes?

\section{L'essoufflement de la créativité dans nos sociétés techniciennes : pour une nouvelle alliance entre sciences " dures", sciences humaines et sociales et savoirs profanes}

L'essoufflement de la créativité dans nos sociétés techniciennes

Avec la chimie de la fin du XVIII ${ }^{\mathrm{e}}$ siècle, le programme cartésienbaconien connaît ses premières réalisations majeures ${ }^{40}$, et au siècle suivant l'industrie chimique est ensuite la première à s'appuyer sur une base véritablement scientifique, inaugurant ainsi un

$\overline{40}$ Dans le Discours de la méthode, Descartes promet que, "grâce à la science, nous deviendrons comme maîtres et possesseurs de la nature ", et au même moment, le chancelier Francis Bacon décrit la réalisation de cette promesse dans la société utopique de La nouvelle Atlantide. Cf. Jean-Marc LévyLeblond, Le grand écart. La science entre technique et culture, Paris, Éditions Manucius, coll. "Modélisations des imaginaires », 2013, p. 18-22. 
couplage de plus en plus étroit entre science et technique ${ }^{41}$. La fin du XIX ${ }^{\mathrm{e}}$ siècle voit apparaître une situation totalement nouvelle sous forme d'une découverte théorique qui ne doit rien à une approche empirique : l'électromagnétisme inventé par Maxwell, alors que les ondes électromagnétiques sont impalpables par nos sens, et donc impossibles à percevoir et à produire sans savoir par avance qu'elles existent - un savoir sans lequel la radio n'aurait jamais pu être inventée. Ce type de couplage va ensuite se généraliser tout au long du $\mathrm{XX}^{\mathrm{e}}$ siècle ${ }^{42}$. Par ailleurs, au XVII e siècle, la science n'est encore qu'une composante de la culture - la "physique " de Galilée reste une branche de la philosophie naturelle ${ }^{43}$. Ce n'est qu'au début du siècle suivant que la science va commencer de s'autonomiser de la culture, dans laquelle elle est née : Diderot, écrivain et philosophe, et D'Alembert, physicien et mathématicien, coéditent la grande Encyclopédie, mais aucun des deux ne comprend vraiment l'autre : le germe d'un écart substitue alors une simple alliance

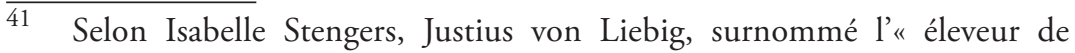
chimistes " du fait d'avoir formé à Giessen, entre 1824 et 1851, des centaines d'étudiants, a fait de la chimie le premier modèle de "science rapide ". Le type de chimiste ainsi formé ne comprend plus les artisans chimistes du siècle précédent, tel Gabriel François Venel caractérisant sa science de "passion de fou » dans l'article «chymie " de l'Encyclopédie, tant il faut l'expérience d'une vie pour acquérir l'art et la maîtrise de la variété subtile et parfois dangereuse des opérations chimiques. Les chimistes formés par Liebig obtiennent désormais un diplôme de doctorat en trois ou quatre ans, sans rien apprendre des recettes et pratiques artisanales, car ils n'ont affaire qu'à des protocoles et à des produits standardisés dans le cadre de la relation quasi symbiotique qui unit désormais la recherche académique et le nouveau réseau des entreprises de chimie industrielle. Cf. Isabelle Stengers, Une autre science est possible! Manifeste pour un ralentissement des sciences, suivi de William James, Le poulpe du doctorat, présenté par Thierry Drumm, Paris, La Découverte, 2013, p. 97 sq.

42 Jean-Marc Lévy-Leblond, $o p$. cit., p. 30-32.

43 Ibid., p. 33-37. Si Galilée peut voir le relief de la Lune dans sa lunette, c'est parce que son oil est exercé par la pratique du dessin, et c'est bien parce que sa maîtrise du clair-obscur et des effets de perspective lui permet de reproduire le relief qu'il est capable de le voir. De même, afin de mesurer le temps que met la bille pour parcourir telle distance sur le plan incliné, Galilée chante, utilisant ainsi la musique comme garde-temps : c'est sa culture musicale qui lui permet de mesurer le temps. 
entre science et culture à la fusion qu'elles connaissaient encore au siècle précédent. $\mathrm{Au} \mathrm{XIX}^{\mathrm{e}}$ siècle, cette coupure s'élargit, et s'institutionnalise toujours davantage avec l'apparition des grandes universités, calquées sur le modèle de l'université de Berlin créée par Humboldt : l'université comme lieu de production du savoir, avec ses propres laboratoires financés sur fonds publics, produit alors une science étroitement couplée à la technique, couplage sur lequel s'appuie le développement du capitalisme industriel. Au $\mathrm{XX}^{\mathrm{e}}$ siècle, enfin, la rupture entre science et culture est consommée : le développement intensif de la science fondamentale est totalement déconnecté du développement culturel tout aussi remarquable qui se manifeste dans la littérature, la peinture, la musique ${ }^{44}$.

Ainsi caractérisée par un couplage massif entre la science et la technique et par un divorce total entre la science et la culture, le développement scientifique de notre époque se trouve dans une situation périlleuse, en ce qu'il risque d'être victime de son propre succès. Car au milieu du XX $\mathrm{X}^{\mathrm{e}}$ siècle s'est opéré un grand changement qui a conditionné toute la suite jusqu'en ce début de $\mathrm{XXI}^{\mathrm{e}}$ siècle : avec le projet Manhattan, la demande technique est entrée directement dans le système de production du savoir, et ce travail sur commande et sous organisation militaro-industrielle a si bien fonctionné - en trois ans, on a fabriqué une bombe atomique qui fonctionne - que les politiques et les militaires ont continué après la guerre de financer abondamment la recherche fondamentale, mais ceci avec une demande de résultats de plus en plus pressante. Tant et si bien que la connaissance scientifique se trouve aujourd'hui arraisonnée par ses propres résultats techniques. Davantage : la technique progresse à nouveau d'une façon de plus en plus autonome, de sorte que de nombreuses découvertes récentes potentiellement utilisables restent scientifiquement incomprises. Cette autonomisation croissante du développement technique et cette marginalisation du développement scientifique sont aujourd'hui telles qu'à l'avenir pourraient

$\overline{44 \quad \text { Ibid., p. 37-41. }}$ 
ne subsister, en guise de sciences fondamentales, que des secteurs peu nombreux et peu coûteux ${ }^{45}$.

L'évolution qui a conduit au type de créativité privilégié par nos prétendues "sociétés de la connaissance " nous a en réalité conduits vers des "économies de la connaissance ${ }^{46}$ " dans lesquelles le contenu concret du terme " connaissance " lui-même a connu une profonde mutation : ce contenu est aujourd'hui celui de savoir-faire englués dans des pratiques dont les fondements scientifiques et épistémiques ne sont plus véritablement interrogés, et qui sont mises au service d'une rentabilité rapide, voire immédiate, dans le cadre d'un pilotage marchand de la recherche. Dans ces conditions, il est devenu très improbable de voir apparaître dans nos sociétés techniciennes des exemples d'analogies créatrices au sens le plus fort, c'est-à-dire sources de ruptures de cadres ou d'apprentissages de niveau 3, telle celle établie par Galilée entre certains phénomènes naturels et certains phénomènes artificiels, amorçant ainsi la méthode expérimentale et la "révolution scientifique » qui s'ensuivit ${ }^{47}$.

45 Ibid., p. 41-49.

46 Cf. Dominique Pestre, Science, argent et politique. Un essai d'interprétation, Paris, INRA Éditions, 2003, et, plus récemment, À contre-science. Politiques et savoirs des sociétés contemporaines, Paris, Seuil, 2013.

47 La généalogie du concept d'onde électromagnétique qui se trouve au cœur de l'électromagnétisme introduit par Maxwell, et donné par Jean-Marc Lévy-Leblond pour le premier exemple d'une découverte théorique qui ne doit rien à une approche empirique (cf. supra, p. 65), offre un exemple fascinant d'une séquence de méta-analogies aboutissant, de Chrysippe à Maxwell en passant par Vitruve, Galilée, Mersenne, Boyle, Newton et Euler, à une telle découverte. Le succès de l'analogie entre les ondes aquatiques (oscillations visibles dans l'eau) et les ondes sonores (oscillations invisibles dans l'air) facilita la méta-analogie entre ces dernières et les ondes lumineuses, dont Maxwell découvrit vers 1860 qu'elles ne reposaient sur le mouvement d'aucun substrat matériel, mais consistaient à l'inverse en des fluctuations périodiques de la magnitude et de la direction de ces entités abstraites que sont les champs électriques et magnétiques. Selon Douglas Hofstadter et Emmanuel Sander, qui développent longuement cet exemple, " les extensions analogiques radicales en physique sont devenues monnaie courante depuis un bon siècle, si bien que la physique théorique est largement devenue l'art de savoir prendre le train de l'analogie en marche, et en particulier de parvenir à trouver le plus prometteur des nombreux trains rivaux - une décision très subtile, et qui se prend, bien sûr, à partir d'analogies avec des trains en marche déjà pris avec 
De fait, il semble bien que les sciences dites « dures » (sciences de la nature, sciences de l'ingénieur, sciences médicales) se trouvent aujourd'hui dans un état d'équilibre stationnaire ${ }^{48}$. Analysé sur une longue période (1900-2004), un corpus très étendu de publications scientifiques montre qu'entre 1955 et 1970 les âges médians (similaires en ce qui concerne les sciences de la nature, les sciences de l'ingénieur et les sciences médicales) des documents cités sont restés stables, et ont augmenté régulièrement depuis cette dernière date, et ceci de manière beaucoup plus significative pour les sciences de la nature et les sciences de l'ingénieur que pour les sciences médicales. Quant à l'âge moyen de la littérature citée, il a diminué par paliers réguliers entre 1945 et 1975, mais a augmenté ensuite tout aussi régulièrement en ce qui concerne les sciences de la nature et les sciences de l'ingénieur, tout en restant plus ou moins stable en matière de sciences médicales. Toutes sciences confondues, les âges médian et moyen de la littérature citée ont donc augmenté depuis le milieu des années 1970, ce phénomène étant toutefois moins accusé en ce qui concerne les sciences médicales.

Contrairement à l'opinion répandue selon laquelle la littérature scientifique serait d'autant plus obsolète qu'elle est âgée, la durée de vie utile de cette littérature a donc globalement augmenté depuis les années 1970 : l'âge moyen des documents cités a augmenté de manière linéaire jusqu’à 1945, puis diminué jusquau milieu des années 1970, mais une troisième période (1980-2004) a vu son retour à une croissance linéaire. Or, les fluctuations de l'âge de la littérature citée constituent un reflet mécanique de celles de la littérature existante, de sorte que ces

succès! Ce jeu intellectuel pourrait être qualifié de "saute-mouton analogique" "(op. cit., p. 262-263). Ce thème est développé par ces auteurs dans le chapitre 8 de leur ouvrage (ibid., p. 527-604), à travers une série d'exemples - notamment celui des découvertes d'Einstein - dont on remarquera qu'ils sont tous très largement antérieurs à la Seconde Guerre mondiale (ibid., p. 527-604).

48 Cf. Vincent Larivière, Éric Archambault et Yves Gingras, "Long-Term Variations in the Aging of Scientific Literature: From Exponential Growth to Staedy-State Science (1900-2004) ", Journal of the American Society for Information Science and Technology, vol. 59, n 2, 2008, p. 288-296. 
résultats indiquent que la recherche scientifique, notamment en ce qui concerne les sciences de la nature et les sciences de l'ingénieur, se trouvent actuellement installées dans un état stationnaire. En définitive, la science dans son ensemble repose actuellement sur un corps de littérature toujours plus âgé : après un âge d'or de la science (1945-1975), où les scientifiques ont résolu beaucoup des problèmes auxquels ils étaient confrontés, aucune "révolution scientifique " majeure n'est apparue, de sorte que la science fonctionne aujourd'hui dans le cadre de la science " normale » au sens de Thomas S. Kuhn ${ }^{49}$.

Cet état stationnaire résulte vraisemblablement en partie du processus amorcé par le projet Manhattan, qui débouche aujourd'hui sur une technoscience où le préfixe " techno " domine très largement le suffixe «science ». Nous voudrions néanmoins compléter ici cette interprétation historico-phénoménologique par une analyse d'ordre sociocognitif, en risquant une hypothèse quant à l'évolution historique de la distribution des choix des acteurs individuels entre communiquer ou cogiter lors de la longue succession d'états du réseau qui a mené à notre modernité à partir de nos racines médiévales.

Communiquer ou cogiter : nous pouvons distinguer ici deux sous-périodes depuis la mutation féodale des $\mathrm{XI}^{\mathrm{e}}-\mathrm{XIII}{ }^{\mathrm{e}}$ siècles. $\mathrm{La}$ première, de loin la plus longue, va de cette mutation jusqu'au milieu des années 1970. Durant ces neuf siècles, il semble que le réseau ait pu sans cesse différer l'état d'équilibre informationnel qui aurait signé sinon son immobilisation finale. Car la mutation féodale a certes débouché sur des communications sociales plus nombreuses et diversifiées que celles qu'avait connues l'époque antérieure, notamment du fait de la montée en puissance du phénomène urbain, mais ces communications sont restées relativement rares en comparaison des flux marquant notre actualité. En d'autres termes, les hommes de ces temps ont vraisemblablement davantage cogité que communiqué, et la richesse productive de leurs processus de cognition internes, notamment via l'analogie

49 Thomas S. Kuhn, The Structure of Scientific Revolutions, Chicago, University of Chicago Press, 1962. 
ou la métaphore, a longtemps pu l'emporter sur la socialisation des catégories existantes via la communication. Certes, parmi l'ensemble de ces analogies et métaphores, toutes n'étaient pas pertinentes, mais sous de multiples formes, nombre d'entre elles ont montré leur fécondité épistémologique, alors même qu'elles n'étaient pas logiquement fondées ${ }^{50}$. La prolifération de catégories mentales qui en résulta a marqué l'évolution sociale et économique des $\mathrm{XII}^{\mathrm{e}}-\mathrm{XX}^{\mathrm{e}}$ siècles européens, caractérisée par un processus ininterrompu d'innovations. Les acteurs principaux en ont d'abord été les artisans, dont les savoirs se sont peu à peu transformés en savoirs d'experts et d'ingénieurs, au rythme même auquel leurs connaissances tacites se sont transformées en savoirs formalisés et codifiés ${ }^{51}$. Alliée à une politique volontariste de l'État en faveur de la formation technicienne, cette évolution a mené, entre 1760 et 1830, à l'épanouissement de la première révolution industrielle en Grande-Bretagne (charbon, machine à vapeur, mécanisation du textile, de la sidérurgie). Dans la société ainsi mise en place, le corpus des savoirs techniques qui s'était construit depuis le début du XIX ${ }^{e}$ siècle s'organisa en filières (mécanique industrielle, hydraulique, etc.) parallèlement à un essor spectaculaire des institutions scientifiques vouées à la recherche, dans les entreprises comme à l'université, et il déboucha dans les années 1880-1914 sur la deuxième révolution industrielle (pétrole et électricité comme nouvelles sources d'énergie, développement de la chimie organique, de l'automobile, etc.) $)^{52}$.

50 Cf. Bernard Ancori, "Analogie, évolution scientifique et réseaux complexes ", op. cit. p. 48 sq. : l'analogie est créatrice au sens fort en faisant fonctionner l'erreur logique consistant à " affirmer le conséquent " dans un syllogisme aristotélicien.

51 Sur les origines artisanales de la révolution scientifique, voir Robert Halleux, Le savoir de la main. Savants et artisans dans l'Europe pré-industrielle, Paris, Armand Colin, 2009, p. 101 sq. Pour sa part, Clifford D. Conner retrace l'apport des savoirs "populaires" aux connaissances scientifiques depuis la préhistoire jusqu’à nos jours. Cf. Clifford D. Conner, Histoire populaire des sciences, Paris, Éditions L'Échappée, 2011 [2005].

52 Nous résumons ici de manière sans doute outrancière les analyses très détaillées de François Caron, La dynamique de l'innovation. Changement technique et changement social (XVI $-X X^{e}$ siècle), Paris, Gallimard, 2010. 
Beaucoup plus courte que la précédente, la seconde période s'ouvre vers le début des années 1970 avec la troisième révolution industrielle - celle des technologies de l'information et de la communication, nées de la rencontre de l'informatique théorique et de la microélectronique. Cette révolution joua très tôt un rôle central au niveau de l'ensemble de nos sociétés en y facilitant à un degré inouï la communication entre les acteurs individuels ou collectifs. Dès 1969, l'Arpanet préfigurait l'Internet; deux ans plus tard, le microprocesseur (Intel) fit son apparition; et Apple commercialisa ses premiers ordinateurs de bureau en 1977. Mais c'est surtout depuis 1995 que ce mouvement s'est accentué avec l'extension exponentielle de l'Internet dans nos sociétés, réputées depuis ce moment précis être fondées sur la connaissance : en 1996, le monde comptait 40 millions d'internautes, et, au milieu de 2011, ils étaient 2,2 milliards, avec une explosion des plateformes portables sans fils (16 millions d'abonnés en 1991, et 5,3 milliards en 2011). Par ailleurs, le nombre d'usagers de réseaux sociaux a surpassé en juillet 2009 le nombre d'usagers d'e-mails ils sont aujourd'hui 1,4 milliards, dont 40 millions en France ${ }^{53}$. Nos sociétés tissées de réseaux voient désormais des acteurs individuels toujours plus nombreux entrer sans cesse en communication ${ }^{54}$, et paraissent ainsi en voie de réaliser l'" homme sans intérieur", cet homme qui serait " tout entier un être communicant ${ }^{55}$ né avec la cybernétique vers le milieu du $\mathrm{XX}^{\mathrm{e}}$ siècle ${ }^{56}$.

53 Cf. Manuel Castells, " Ni dieu ni maître : les réseaux ", Fondation Maison des Sciences de l'Homme, Working Paper 02, février 2012.

54 Manuel Castells, La société en réseaux. L’ère de l'information, Paris, Fayard, 1998.

55 Philippe Breton, "La représentation de l'humain et la construction d'une image de l'homme "sans intérieur" ", dans Dominique Weil (dir.), Figures du sujet dans la modernité, Strasbourg, Éditions Arcanes, 1997, p. 229-242.

56 La cybernétique est née avec l'article classique d'Arturo Rosenblueth, Norbert Wiener et Julian Bigelow, "Behavior, Purpose and Teleology ", Philosophy of Science, vol. X, 1943, p. 18-24. L'un des objectifs de cet article était alors de promouvoir la notion de comportement. Cinq ans plus tard, Norbert Wiener substitua à ce terme celui de communication, dont il prétendait que la cybernétique était la science (cf. Norbert Wiener, Cybernetics, or Control and Communication in the Animal and the Machine, Cambridge (MA), MIT Press, 
En définitive, après que la richesse productive de l'analogie et de la métaphore a longtemps pu l'emporter dans nos sociétés sur la socialisation des catégories existantes par la communication, il semble que notre modernité se trouve aujourd'hui dans le cas inverse $\mathrm{du}$ fait de conditions techniques privilégiant toujours davantage la communication au détriment de la cognition ${ }^{57}$. Toujours davantage, car nous savons que ce processus est cumulatif, de sorte que le réseau devrait se diriger à un rythme accéléré vers un état final consistant en un amas unique d'acteurs au sein duquel tous seraient strictement identiques. Toute créativité faible liée à la communication ayant alors disparu, aucune créativité forte associée à la cognition ne pourrait plus subsister. La trajectoire convergeant vers cette forme de mort entropique du réseau apparaît aujourd'hui ponctuée par une succession d'états transitoires marqués par la formation d'amas locaux d'acteurs, et la différenciation progressive de ces amas sous forme d'une " tribalisation du monde ${ }^{58}$ " est corrélative d'une diminution continue de la créativité globale du réseau.

Il en va de même dans le cadre restreint du seul monde académique, dont l'état stationnaire actuel en matière de productions

1948). Sur l'histoire de la cybernétique, voir Jérôme Segal, Le Zéro et le Un. Histoire de la notion scientifique d'information au XXe siècle, Paris, Editions Syllepse, 2003, et Mathieu Triclot, Le moment cybernétique. La constitution de la notion d'information, Paris, Éditions Champ Vallon, 2008. Pour une interprétation de l'impact de la cybernétique, et du paradigme communicationnel qu'elle véhicule, sur les sciences sociales du XX siècle, voir Cécile Lafontaine, L'empire cybernétique. Des machines à penser à la pensée machine, Paris, Seuil, 2004.

57 Notons que François Caron définit l'innovation comme « un processus de recomposition des savoirs existants " dans Les voies de l'innovation: les leçons de l'histoire, Paris, Éditions Manucius, coll. "Modélisations des imaginaires ", 2011, p. 9. Dans notre perspective, cela signifie que l'historien de l'innovation considère actuellement celle-ci comme le résultat de la créativité faible liée à la communication entre les acteurs, plutôt qu'à la créativité forte associée à la cognition productrice d'analogies et de métaphores inédites. Cf. Michel Maffesoli, Le Temps des tribus. Le déclin de l'individualisme dans les sociétés de masse, Paris, Librairie des Méridiens, Klincksieck et Cie, 1988, et La Transfiguration du Politique. La tribalisation du monde, Paris, Grasset et Fasquelle, 1992, ainsi que Michel Maffesoli et Brice Perrier, L'homme postmoderne, Paris, François Bourin Éditeur, 2012. 
en sciences « dures » pourrait tenir au très large privilège conféré par nos sociétés techniciennes à la communication par rapport à la cognition. La technique intervient en effet doublement dans un processus de mutation de la notion même de créativité. D'une part, elle substitue globalement son épistémologie propre à celle de la science fondamentale et pousse cette substitution jusqu'à inverser le projet cartésien en celui qui se propose aujourd'hui - via les nanosciences et la biologie synthétique - de fabriquer la nature pour mieux la comprendre ${ }^{59}$. Et d'autre part, les facilités offertes par la révolution des technologies de l'information et de la communication accentuent toujours davantage le privilège donné à la communication au détriment de la cognition. Mais surtout, au-delà d'une mutation, cette évolution se traduit également par une perte collective de créativité, car là où l'analogie et la métaphore permettent parfois de "sortir du cadre " (thinking out of the box), c'est-à-dire de changer de système de pensée plutôt que de se contenter de modifier les éléments d'un système de pensée en lui-même inchangé ${ }^{60}$, la communication non stop vient au contraire consolider toujours davantage le cadre technicomarchand existant à la faveur des apprentissages secondaires auxquels elle donne lieu.

En effet, ainsi que Gregory Bateson nous l'a appris en systématisant une idée qu'il disait tenir de Samuel Butler, plus nous savons quelque chose, moins nous sommes conscients de ce savoir : il existe un processus par lequel le renforcement continu de l'apprentissage secondaire par itérations successives d'apprentissages primaires d'un certain type aboutit à enfouir dans les profondeurs du non-conscient les cadres de l'expérience alors acquise ${ }^{61}$. À la limite, l'apprentissage secondaire ainsi réalisé se transforme en une routine cognitive parfaitement ancrée. Sur le plan socio-

59 Cf. Bernadette Bensaude-Vincent, Les vertiges de la technoscience. Façonner le monde atome par atome, Paris, La Découverte, 2009; Marie-Hélène Parizeau, Biotechnologie, nanotechnologie, écologie. Entre science et idéologie, Versailles, Éditions Quae, 2010; Bernadette Bensaude-Vincent et Dorothée BenoitBrowaeys, Fabriquer la vie. Où va la biologie de synthèse?, Paris, Seuil, 2011.

60 Luc de Brabandere, Pensée magique, pensée logique. Petite philosophie de la créativité, Paris, Éditions du Pommier, 2012.

61 Gregory Bateson, Steps to an Ecology of Mind, op. cit. 
politique, ce type de routine s'apparente à une idéologie - au sens péjoratif qu'a pris ce terme - d'autant plus pernicieuse qu'elle agit souvent à l'insu total de ceux qui mettent en actes les injonctions qu'elle véhicule. Telle nous apparaît aujourd'hui l'idéologie technico-marchande associée à l'injonction permanente à communiquer qui ne cesse de la conforter - une injonction rapidement radicalisée en un impératif dont la généalogie et les effets délétères furent reconnus et analysés dès la fin des années $1980^{62}$. Ces analyses n'ont malheureusement guère abouti à stopper, ni même à ralentir, une évolution qui semble aujourd'hui irrépressible ${ }^{63}$, et qui a introduit l'idéologie de la communication jusqu'au cœur de la partie de nos sociétés dont la vocation proclamée est pourtant de se tenir à distance de toute idéologie par la distance critique qu'elle se flatte de cultiver : le monde académique.

Pour une nouvelle alliance entre sciences "dures ", sciences humaines et sociales et savoirs profanes

Sans être universelle, l'emprise grandissante de l'idéologie de la communication est devenue suffisamment massive dans le monde académique - sous forme d'un publish or perish omniprésent pour qu'il soit très largement illusoire de la voir être combattue, et encore moins se dissiper, à partir de l'intérieur même de celuici, qu'il s'agisse des sciences "dures " ou des sciences humaines et sociales auxquelles les précédentes réussissent de mieux en mieux à imposer leur modèle ${ }^{64}$. En particulier, il ne faut guère

62 Philippe Breton et Serge Proulx, L'explosion de la communication, Paris, La Découverte, 1989; Lucien Sfez, Critique de la communication, Paris, Seuil, 1989.

63 Il est significatif que, vingt ans après la publication de ces ouvrages critiques, l'un de leurs auteurs se soit senti tenu de publier un dialogue en forme de manifeste contre les excès de la communication. Cf. Philippe Breton et David Le Breton, Le silence et la parole. Contre les excès de la communication, Toulouse, Éditions Érès, 2009.

64 D'où les faibles chances de succès, selon nous, d'un appel tous azimuts (notamment sous forme de pétitions) à une slow science à laquelle se convertiraient spontanément les scientifiques. Sans illusions à ce sujet, Isabelle Stengers, "Une autre science est possible... ", op. cit., analyse avec conviction les caractéristiques de cette slow science sous forme de rapports 
compter pour cela sur les jeunes générations, qui sont pour ainsi dire nées dans cette idéologie et en acceptent apparemment d'autant plus volontiers les règles qu'elles semblent juger ces dernières aussi incontournables que des lois naturelles de portée universelle ${ }^{65}$. Dans notre visée théorique soucieuse de cohérence avec les données observables, ce n'est donc pas l'introduction de générations successives d'acteurs académiques qui pourrait venir contrecarrer l'évolution de leur monde vers l'homogénéisation des représentations qu'y provoque toujours davantage la communication.

Que faire? La croissance de l'entropie n'étant irréversible qu'au sein de systèmes clos, d'un point de vue théorique il convient certes d'ouvrir notre réseau d'acteurs académiques, mais ceci dans l'espace plutôt que dans le temps : une solution possible passe ici par une ouverture systématique et efficace des représentations des acteurs du monde académique à d'autres représentations d'autres acteurs provenant d'autres mondes. Car ce type d'ouverture pourrait être l'occasion de confrontations fécondes entre les cadres primaires respectifs des différents mondes en présence, et ces confrontations seraient alors elles-mêmes susceptibles de provoquer d'heureuses ruptures de cadres dans les représentations de chacun des acteurs peuplant ces mondes ${ }^{66}$. Certes, il ne faut guère attendre de telles ruptures qu'elles affaiblissent l'idéologie de la communication, puisque celle-ci est identiquement répandue dans l'ensemble de la société. Il ne sagit donc pas de moins communiquer pour cogiter davantage, mais plutôt de cogiter autrement grâce à l'établissement de communications inédites entre des

entièrement à repenser entre sciences de la nature, sciences humaines et sociales et " connaisseurs avertis" (cf. infra).

65 Après avoir souligné la mobilisation relativement faible des générations actuelles de chercheurs contre la flexibilité et la compétition imposées à tous par l'économie de la connaissance sous peine d'être éliminés dans la course à la carrière, Isabelle Stengers conclut sombrement : "On peut prévoir que la génération des chercheurs qui vient sourira avec cynisme lorsqu'on évoquera l'heureux temps où les chercheurs posaient leurs propres questions » (ibid., p. 44).

66 Sur le rôle que peuvent jouer les " profanes " à cet égard, voir par exemple Frédéric Naudon, "Comment le profane joue en faveur du décloisonnement ", Hermès, n 67, CNRS Éditions, 2013, p. 62-67. 
amas d'acteurs qui actuellement s'ignorent très largement. C'est alors par le renouvellement des points de vue et des modes de cognition issu de la mise en présence systématique d'acteurs composant ces amas différents que peuvent apparaître de nouvelles catégories mentales et de nouvelles combinaisons de telles catégories, synonymes d'un sursaut de créativité propre à différer la mort entropique du réseau.

Ce type d'ouverture et de confrontation constitue la condition sine qua non d'une rupture de cadre dans les représentations d'acteurs de sciences " dures " majoritairement engluées dans le type de "science rapide " inauguré au XIX siècle par la chimie de Liebig, et dont le mode de fonctionnement domine aujourd'hui l'orientation technico-marchande que ce type de science a progressivement privilégiée au détriment de la science fondamentale et des sciences humaines et sociales ${ }^{67}$. Selon Isabelle Stengers, ce mode de fonctionnement a pour caractéristique centrale de fabriquer des " chercheurs somnambules » dont les stratégies de recherche rappellent la "parabole du réverbère " en consistant à rechercher des clés perdues aux seuls endroits éclairés. Formatés à ne se poser que les questions dont ils pensent qu'elles présenteront un intérêt pour quiconque sera susceptible de valoriser les réponses qu'ils leur auront données, et rejetant dans les ténèbres de l'« opinion " toutes les autres, ces chercheurs somnambules s'identifient au cerveau pensant et rationnel de l'humanité et estiment que seul leur point de vue détermine légitimement la manière dont tel ou tel problème doit être posé. Ce genre de formatage est d'autant plus efficace qu'il procède de la force du paradigme, qui est d'être invisible selon Thomas S. Kuhn, de sorte que cette "bonne " manière de poser les questions relève, pour les esprits ainsi formatés, de l'évidence incontestable et

67 Comme le note Isabelle Stengers, « [n] ous touchons ici le caractère remarquablement asymétrique des savoirs produits sous le modèle de la science rapide. Les techniques dites "matérielles" ont explosé, mais celles que l'on dit "immatérielles" ou "humaines" se sont plutôt appauvries " (op. cit., p. 110). 
incontestée que nous savons être celle de routines cognitives parfaitement ancrées ${ }^{68}$.

Contre l'emprise grandissante de cette "science rapide ", le manifeste d'Isabelle Stengers prône un ralentissement des sciences adressé aux scientifiques eux-mêmes. Le type de slow science ainsi promu prendrait le contre-pied exact des caractéristiques brièvement rapportées ci-dessus. Le modèle en serait celui d'une " consultation " portant sur une matter of concern intéressant l'ensemble des citoyens, et dont le propre serait d'exclure l'idée de la bonne solution incarnée par telle ou telle innovation que la propagande et souvent l'expertise scientifiques privilégient trop souvent au nom de la science ${ }^{69}$. Ce type de consultation rassemblerait et mêlerait scientifiques professionnels (sciences "dures" et sciences humaines et sociales confondues) et l'" amatorat distribué » de " connaisseurs avertis " au sein d'une constellation de "savoirs situés " sur un mode qui en interdirait l'appropriation au nom de quelque idéal abstrait que ce soit ${ }^{70}$.

Les scientifiques, notamment, devraient abandonner l'illusion de détenir un point de vue transcendant lié à une supposée position de surplomb, en présentant désormais leur savoir comme partie prenante parmi d'autres de chaque situation problématique, sans prétendre au moindre privilège quant à la manière dont le problème est formulé et dont ses solutions sont envisagées $^{71}$. En définitive, ce processus demanderait "que nul ne puisse être autorisé à définir "ce qui importe vraiment" 72 ", et instaurerait ainsi un nouveau type de "civilité " où chaque consultation exigerait un co-apprentissage transformant le savoir de chacun en le dépouillant activement des économies de pensée qui lui donnent raison contre les autres, rendant ceux qui se réunissent capables de penser et d'imaginer ensemble ${ }^{73}$.

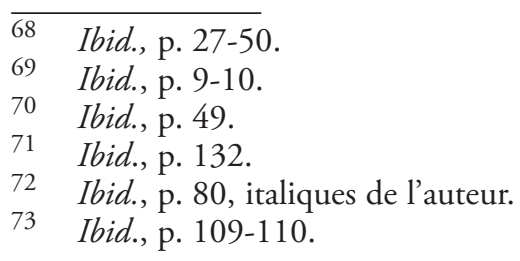


Une slow science ouverte à tous les points de vue et questionnements placés sur un pied d'égalité contre une "science rapide " appropriée par les seuls scientifiques dont le point de vue dominerait tous les autres, et dont les «bonnes " questions continueraient de rejeter toute autre forme d'interrogation dans les ténèbres de l'opinion : une telle situation est très exactement celle d'une confrontation entre deux conventions, dont l'une (minoritaire, car émergente) voudrait supplanter l'autre (majoritaire, donc installée) au sein d'une population donnée d'acteurs ${ }^{74}$. Certains aspects de la théorie lewisienne des conventions vont donc à présent nous permettre de définir une modalité efficace de l'ouverture du monde académique à d'autres mondes que représenterait l'avènement de la slow science prônée par Isabelle Stengers ${ }^{75}$.

La notion de convention a été introduite par David Lewis afin de lever l'indétermination subsistant entre plusieurs équilibres de Nash dans un « jeu de coordination » à la Thomas C. Schelling ${ }^{76}$ : le choix entre ces équilibres possibles étant indécidable par le seul

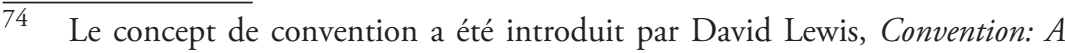
Philosophical Study, Cambridge (MA), Harvard University Press, 1969. Depuis sa parution, cet ouvrage a suscité une littérature considérable dont nous sélectionnons ici les seuls aspects directement pertinents pour notre propos : sur la généalogie de l'ouvrage fondateur de David Lewis et ses prolongements immédiats, voir Éléonore Le Jallé, « La convention : ce que Lewis doit (ou non) à Hume ", Klesis - Revue philosophique, n 24, 2012, p. 239271; sur ses développements en sciences économiques et de gestion, voir André Orléan (dir.), Analyse économique des conventions, Paris, Presses Universitaires de France, 1994, Pierre-Yves Gomez, Qualité et Théorie des Conventions, Paris, Economica, 1994, Philippe Batifoulier, Théorie des conventions, Paris, Economica, 2001; pour une analyse sociocognitive de la genèse des conventions, voir Bernard Ancori, "Réseaux complexes et genèse des conventions ", 6 Congrès Européen des Sciences des Systèmes, Paris, 19-22 septembre 2005; enfin, pour une critique de l'économie des conventions, notamment de son individualisme méthodologique et de la prépondérance qu'elle donnerait aux dimensions cognitives de la réalité, voir Bruno Amable et Stefano Palombarini, L'économie politique n'est pas une science morale, Paris, Raisons d'Agir Éditions, 2005.

75 Isabelle Stengers (op. cit., p. 61 sq.) utilise également le terme " convention ", mais ceci dans un tout autre sens que celui introduit par David Lewis.

76 Thomas C. Schelling, The Strategy of Conflict, Oxford, Oxford University Press, 1960. 
calcul individuel, et cette indétermination pouvant précipiter les joueurs vers des solutions désastreuses, seul un mode de coordination collective peut dénouer la situation en sélectionnant l'un de ces équilibres ${ }^{77}$. Ce mode de coordination collective est une convention, qui apparaît ainsi comme un comportement standard que l'agent individuel est rationnellement conduit à observer plutôt que de s'en écarter de manière isolée. David K. Lewis a ensuite complété et précisé sa définition : une convention est une régularité $\mathrm{R}$ de comportement (et/ou de croyance) qui, dans une population $\mathrm{P}$, satisfait à six conditions : 1) chacun se conforme à $\mathrm{R}$; 2) chacun croit que les autres se conforment à R; 3) cette croyance incite chacun à se conformer effectivement à $\mathrm{R}$; 4) tous préfèrent une conformité générale à $\mathrm{R}$ plutôt que moins que générale (en particulier qu'une conformité de tous sauf un); 5) il existe au moins une autre régularité $\mathrm{R}^{\prime}$ qui satisfait les conditions 3 et 4 ; 6) les cinq conditions précédentes sont common knowledge $e^{78}$.

Soient R la convention "science rapide ", et R' la convention slow science. À quelles conditions la convention R' peut-elle supplanter la convention R? C'est précisément à cette question que répondent Robert Boyer et André Orléan en proposant deux modèles successifs de dynamiques des conventions : l'effondrement et l'accord ${ }^{79}$. Ces modèles supposent tous deux qu'une convention est une stratégie évolutionnairement stable, au sens de John Maynard Smith dans un jeu qui en comporte au moins deux : une stratégie telle que, si elle est suivie par tous les

77 Un exemple souvent cité dans la littérature à la suite de Thomas C. Schelling est celui de la conduite automobile. Il existe ici deux équilibres de Nash (tout le monde roule à gauche, ou tout le monde roule à droite), et le calcul individuel ne fournit aucune raison de privilégier l'un ou l'autre. De sorte que les automobilistes risquent de se précipiter vers une solution désastreuse, consistant à rouler à droite pour une partie d'entre eux, et à gauche pour l'autre partie.

78 David K. Lewis, "Languages and Language ", Philosophical Papers, vol. I, Oxford University Press, 1983, p. 165-166.

79 Robert Boyer et André Orléan, « Persistance et changement des conventions. Deux modèles simples et quelques illustrations ", dans André Orléan (dir.), Analyse économique des conventions, Paris, Presses Universitaires de France, 1994, p. 219-247. 
membres d'une population, alors aucune stratégie mutante ne peut envahir cette population ${ }^{80}$. Cette définition de la convention permet d'exprimer son caractère auto-renforçant et partiellement arbitraire, ainsi que de montrer que la population concernée peut se trouver bloquée dans une convention non Pareto-optimale : bien que la convention mutante puisse être Pareto-supérieure à la convention existante, cette dernière se maintient. Le modèle de l'effondrement montre alors que, lorsque la convention $\mathrm{R}$ prévaut, on ne peut évoluer vers R' que si la proportion de ceux qui adoptent $\mathrm{R}^{\prime}$ dépasse un certain seuil critique. Ce seuil fonctionne donc comme une «barrière de potentiel » devant être franchie pour que l'ensemble de la population converge vers la convention R' - auquel cas la convention $\mathrm{R}$ s'effondre.

Au contraire de cette dynamique où l'atomisation des individus leur interdit de communiquer entre eux, le modèle de l'accord s'appuie sur la richesse des procédures explicites de concertation et d'harmonisation des stratégies individuelles qui existent dans nos sociétés. Ainsi des acteurs constatant le blocage de l'ensemble de leur population dans une convention non Pareto-optimale peuvent s'accorder sur des procédures socialement construites en vue d'établir une convention associée à une utilité supérieure. Nous sommes donc typiquement dans une situation où les partisans de la slow science constateraient la perte de créativité liée à la domination de la "science rapide ", et voudraient en conséquence la détrôner en tant que convention dominante. Sur le plan analytique, la possibilité de communication entre ces partisans d'une slow science au sein de réseaux sociaux, lieux d'une diffusion endogène de leur convention jugée supérieure, se traduit par une localisation des interactions dans la population globale. Par contraste avec les acteurs évoqués dans le cadre de l'effondrement, parmi lesquels ceux qui choisissaient une stratégie minoritaire R' rencontraient principalement des acteurs ayant choisi la stratégie majoritaire $\mathrm{R}$ et obtenaient ainsi une utilité très faible, les acteurs choisissant R', à présent impliqués dans une

80 John Maynard Smith, Evolution and the Theory of Games, Cambridge, Cambridge University Press, 1982. 
dynamique d'accord, se reconnaissent entre eux et augmentent leurs probabilités d'interagir avec d'autres acteurs ayant également choisi R'.

Avec une formalisation différente de la nôtre, mais dont l'esprit est très proche de celle-ci, Robert Boyer et André Orléan démontrent alors un résultat fondamental pour notre propos : plus les interactions sont localisées, plus la "barrière de potentiel " introduite dans le modèle de l'effondrement est abaissée, et plus grandes sont les possibilités de diffusion pour le groupe innovant. Lorsque la localisation des interactions est minimale, l'interaction étant totalement indifférenciée comme dans le modèle de l'effondrement, la nouvelle convention R' ne peut pas se diffuser, et l'ancienne convention $\mathrm{R}$ se maintient. Mais lorsque le degré de localisation des interactions est maximal, c'est-à-dire lorsque chaque acteur n'interagit qu'avec ses deux voisins immédiats comme dans notre propre modèle, la condition suffisante de diffusion de la nouvelle convention R' est que son utilité soit supérieure à celle de l'ancienne convention R. Pour tous les degrés intermédiaires de localisation des interactions, il existe une valeur - toujours inférieure à celle qui correspond à la "barrière de potentiel » du modèle de l'effondrement - telle que si la taille du groupe innovant lui est supérieure, alors la nouvelle convention se diffuse. Robert Boyer et André Orléan en déduisent finalement qu'une convention installée et désireuse de se maintenir a toujours intérêt à imposer aux conventions concurrentes une contrainte d'universalité immédiate. Inversement, la nouvelle convention ne doit chercher qu'à s'implanter progressivement ${ }^{81}$.

$\overline{81}$ Robert Boyer et André Orléan, « Persistance et changement des conventions...", op. cit., p. 236, nos italiques. L'exemple des conventions salariales dans les transplants japonais aux États-Unis et au Royaume-Uni illustre parfaitement cette démonstration (ibid., p. 237-243). Et nous pouvons également comprendre en ces termes l'échec de l'acclimatation du denier carolingien dans l'économie morcelée de la Gaule franque : c'était alors la nouvelle convention, soutenue par les Carolingiens à la suite de Charlemagne, qui prétendait imposer une contrainte d'universalité, alors que les conventions existantes (les consensus monétaires partiels, dont la juxtaposition était héritée des Mérovingiens) s'appuyaient au contraire sur des réseaux d'interactions localisées. Cf. Bernard Ancori, Échange monétaire et Évolution économique, Thèse de Doctorat d'État ès-Sciences économiques, Université 
Ces deux modèles de dynamiques des conventions nous permettent de donner un contenu précis à la notion d'efficacité d'une modalité d'ouverture systématique du monde académique à d'autres mondes, telle celle que représenterait l'avènement $\mathrm{du}$ type de slow science prôné par Isabelle Stengers. En effet, parmi la grosse cinquantaine de dispositifs différents qui se réclament d'une démocratie participative à travers le monde, et pourraient ainsi contribuer à cette ouverture systématique, celui qui remplit au mieux les conditions pour que cette dernière soit efficace est incarné par le type de forum hybride introduit par Michel Callon, Pierre Lascoumes et Yannick Barthe. Car la nouvelle convention portée par ce type de forums opère par définition au sein de réseaux localisés, et se trouve donc à l'exact opposé de toute contrainte d'universalité immédiate. Organisés en principe à l'initiative de tout citoyen ou groupe de citoyens concerné(s) par une question sociotechnique controversée, ces forums possèdent ainsi sui generis une dimension concrète et localement ancrée qui les situe également à mille lieues des grands débats sur des thèmes très (trop) généraux organisés à l'attention d'un " grand public " dont la notion même est problématique. Enfin, lors de ces forums qui se déroulent dans l'espace public, tous les points de vue sont a priori également légitimes dès lors qu'ils sont rationnellement argumentés : investis en tant que lieux de paroles (ce sont bien des forums) par des acteurs concernés à des titres divers par les sujets en question (scientifiques, associations, syndicats, entrepreneurs, décideurs politiques, mais aussi individus lambda), ils constituent des lieux d'exploration collective des aspects scientifiques, techniques, culturels, économiques, politiques et éthiques de ces sujets (ces forums sont bien hybrides). Contrastant avec une recherche "confinée " menée dans le secret des laboratoires, ces forums inventent ainsi une recherche " en plein air " dans le cadre d'une démocratie "dialogique ». Dans ce cadre, l'ensemble des parties prenantes identifie collectivement la nature

Louis Pasteur (Strasbourg I), 1990, p. 72-81, et «Évolution, complexité et consensus monétaire : un modèle théorique et quelques illustrations historiques ", Économie Appliquée, Tome L, n 3, 1997, p. 224-226. 
même des questions à traiter, construit ses propres modes de fonctionnement et d'échanges, et débat des solutions possibles au cours d'un processus d'apprentissage sans limite assignée $a$ priori $^{82}$.

Dans ce type de dispositif, la modalité d'échanges privilégiée est la controverse, qui offre à toutes les parties prenantes un mode d'exploration privilégié des groupes concernés, des problèmes posés et de leurs solutions possibles. Ces forums sont ainsi le lieu d'une révélation simultanée des différentes facettes de ces problèmes, parfois totalement ignorées des experts académiques, de sorte que les controverses qui s'y déroulent s'avèrent constituer de puissants outils d'apprentissages collectifs, en faisant circuler l'information entre groupes ou acteurs comme en permettant la mise en commun de leurs savoirs. Cette mise en commun ne signifie nullement que les "profanes " auraient à s'approprier la culture technique des "savants ", et elle ne signifie pas davantage que les "savants" auraient simplement à tenir compte des avis des profanes dans la gestion de risques qu'ils auraient auparavant été seuls à pouvoir évaluer. Elle doit bien plutôt être conçue comme un assemblage épistémique dont toutes les facettes sont également offertes à l'appréciation de chacun des acteurs. Les parties prenantes procèdent ainsi collectivement à une évaluation des risques toujours révisable, au terme toujours provisoire d'un nouvel apprentissage. Cette évaluation dépend de l'identification des mondes possibles dont ce processus d'apprentissage favorise l'émergence, et ce n'est que lorsque ce processus débouche sur une stabilisation jugée satisfaisante de cet éventail de mondes possibles et des risques qui leur sont associés qu'advient le moment de la décision. Issue de cette dernière, la gestion des risques prend alors la forme de " lois expérimentales », qui entrent en vigueur pour une durée déterminée au terme de laquelle elles se voient réévaluées ${ }^{83}$.

82 Michel Callon, Pierre Lascoume et Yannick Barthe, Agir dans un monde incertain. Essai sur la démocratie technique, Paris, Seuil, 2001.

83 Michel Callon et Yannick Barthe, «Décider sans trancher. Négociations et délibérations à l'heure de la démocratie dialogique ", Négociations, n 2 , 2005, p. 115-129. 
En définitive, l'objectif d'un forum hybride se situe simultanément à deux niveaux différents et intimement liés. À un premier niveau, il consiste à instaurer parmi ses membres un processus d'apprentissage collectif à propos d'une question sociotechnique précise et controversée. Il rassemble ainsi les représentations de l'ensemble de ses membres dans un réseau émergent de savoirs appropriables, rivaux et spécifiques ${ }^{84}$. À un second niveau, l'objectif du forum hybride consiste en la recherche de "bonnes pratiques" en matière de co-construction de bases consensuelles minimales permettant des prises de décisions robustes sur des sujets controversés. Il s'agit alors de donner un caractère transférable aux enseignements acquis tout au long du processus d'apprentissage collectif qu'il favorise, et de transformer ainsi le réseau émergent en lequel consiste initialement le forum en un réseau métastable. En d'autres termes, sans aller jusqu’à figurer un réseau totalement stabilisé au sein duquel les représentations des acteurs seraient devenues des savoirs non appropriables, non rivaux et généraux, il s'agit pour le forum d'évoluer vers un réseau dont les membres seraient porteurs et vecteurs de savoirs capitalisés et communicables relativement facilement et à coût faible au-delà du réseau. Une telle évolution débouche ainsi sur une montée en généralité, et les réseaux localisés que constituent chacun de ces forums peuvent alors être interconnectés, de sorte que la convention slow science dont ils sont adhérents peut supplanter la convention «science rapide » jusque là dominante au sein de la population globale.

Nous débouchons ainsi sur une version plus compliquée des deux dilemmes exposés au début de ce texte. Certes, comme le réseau sociocognitif complexe d'acteurs individuels hétérogènes dont la structure et l'évolution la plus probable ont été évoquées ci-dessus, un forum hybride doit traiter le dilemme " créativitél proximité » de manière à assurer en permanence un réglage fin visant à assurer la meilleure productivité possible de la commu-

84 Michel Callon, «Le réseau comme forme émergente et comme modalité de coordination : le cas des interactions stratégiques entre firmes industrielles er laboratoires académiques ", dans Michel Callon et al., Réseau et coordination, Paris, Economica, 1999, p. 13-64. 
nication. Il se trouve ainsi dans une situation analogue à celle d'une firme désireuse de s'allier à des laboratoires de recherche, "détenteurs de compétences et de savoir-faire suffisamment différents des siens pour lui permettre l'ouverture de nouvelles options technologiques, mais néanmoins suffisamment proches pour que la communication soit possible et point trop coûteuse ${ }^{85}$ ".

Mais, à l'instar d'une telle firme adhérant à la convention "science rapide ", le forum adhérant à la convention slow science constitue une illustration concrète du réseau très abstrait analysé précédemment, et nous ne pouvons pas le considérer comme un réseau d'acteurs strictement individuels, car il s'agit en réalité d'un réseau de réseaux : certains de ses membres sont membres d'une institution - association, filière professionnelle, collectivité territoriale, université ou organisme de recherche, communauté de voisins - et chacune de ces institutions est elle-même un réseau. D'où une inéliminable ambiguïté en matière d'identités des acteurs impliqués : sont-ils là à titre individuel ou en tant que porte-parole de leurs institutions? Comment cette identité évolue-t-elle au fil des interactions successivement nouées, au sein de ce réseau de réseaux comme en celui de chacun de ces derniers? Le problème se complique encore du fait qu'un forum hybride est par construction ouvert en permanence à toute partie intéressée par la ou les question(s) qui lui donnent sens et existence. À l'incessante redéfinition des identités des acteurs déjà membres du réseau, provoquée par les processus d'apprentissage qu'ils réalisent dans ce cadre, s'ajoute donc une série de difficultés liées à l'entrée toujours possible de nouveaux acteurs. Car cette entrée modifie en permanence les données du problème, en déformant ipso facto la distribution préalable des proximités cognitives donc des propensions à communiquer - parmi les membres du réseau. Le dilemme "créativité/proximité " se pose donc ici dans le cadre dynamique d'un réseau de réseaux dont la démographie n'est jamais définitivement fixée, et sa résolution est autrement

85

Michel Callon, op. cit. p. 56. 
plus complexe que dans le cas relativement simple d'un réseau d'individus dont la population serait donnée.

Enfin, une autre difficulté vient plutôt impacter ici le dilemme " communication/cognition " : celle du choix entre l'accueil, au sein d'un réseau constitué, de nouveaux acteurs plutôt proches ou plutôt éloignés, du point de vue de leurs univers cognitifs, de ceux déjà présents dans le réseau. En effet, plus les nouveaux acteurs sont proches des anciens, plus ils auront tendance à communiquer avec eux plutôt qu'à cogiter, et inversement. Le choix dépendra donc d'une évaluation de la situation du dilemme "communication/cognition " au moment de l'entrée des nouveaux : si cette situation est jugée trop favorable à la communication, il conviendra de favoriser l'entrée d'acteurs dont les univers cognitifs sont plutôt hétérodoxes, et inversement. Et ce choix influera nécessairement sur les rapports de force entre les acteurs du réseau ${ }^{86}$. En pratique, nous pouvons considérer que chaque réunion du forum hybride est occasion de communiquer

Dans un travail antérieur, nous avons montré qu'au sein d'un réseau constitué de deux réseaux concurrents et qui pèsent d'un poids égal sur le réseau global, l'adjonction d'un nouveau membre à l'un de ces deux réseaux ne lui assurait pas nécessairement un poids supérieur à celui de l'autre, car il faut également prendre en compte les degrés de proximité respectifs du nouvel entrant avec les membres de ces deux réseaux dans l'emprise relative de chacun de ces derniers : s'il est trop dissemblable des membres du réseau dans lequel il entre, ce nouvel entrant vient en réalité l'affaiblir face à l'autre réseau. Pour prendre un exemple dans un domaine où, toutes choses égales par ailleurs, le facteur démographique devait être plus déterminant que dans d'autres - celui de la guerre antique -, les soldats-citoyens de la petite cité athénienne n'auraient jamais pu sinon vaincre à Marathon (en 490 av. J.-C.) l'immense armée de l'empire perse envoyée par Darius lors de la première guerre Médique. Cf. Bernard Ancori, "Controverses et consensus scientifiques et sociotechniques entre théorie des consensus et sémantique des mondes possibles ou Glissements progressifs des sciences économiques vers la logique formelle. Petit exercice de réflexivité sous forme de témoignage sur une pratique de formalisation dans la construction d'une épistémologie ", Séminaire de l'Institut de Recherches Interdisciplinaires sur les Sciences et la Technologie, (IRIST EA 3424), Strasbourg, 24 novembre 2006. La victoire de Marathon fut essentiellement due à la discipline des hoplites grecs organisés en phalange face à une armée perse composée d'esclaves indisciplinés. Or, la caractéristique centrale de la phalange hoplitique était la solidarité entre ses éléments parfaitement interchangeables du fait de leur parfaite similitude. Cf. Marcel Détienne, Les Maîtres de Vérité dans la Grèce archaïque, Paris, Maspéro, 1967. 
pour ses membres, alors que l'intervalle temporel séparant deux réunions successives est plutôt dédié à leurs processus cognitifs. La solution du dilemme " communication/cognition " passe donc ici par une ponctuation judicieuse des réunions du forum dans le planning global de ses activités.

Ces difficultés n'obèrent en rien le fait que l'avènement d'une slow science en lieu et place de la " science rapide " qui domine nos sociétés techniciennes y serait synonyme d'un surcroît de créativité mais aussi de rationalité, comme le montre l'exemple du Comité local de suivi de l'INRA-Colmar qui fonctionne sur le mode d'un forum hybride depuis 2003, et dont certaines questions portées par ses membres " profanes » ont pu déboucher sur un véritable renouvellement des interrogations et des programmes de recherche de ses membres académiques ${ }^{87}$.

87 Anne Moneyron, Olivier Lemaire et Jean Masson, «Une expérience d’interface science/société. Consolider la recherche en s'appuyant sur les savoirs profanes, la complexité et l'engagement sur le temps long ", Futuribles, mars, $\mathrm{n}^{\circ} 383,2012$, p. 111-118; The Local Monitoring Committee, "Interactive Technology Assessment and Beyond: the Field Trial of Genetically Modified Grapevines at INRA Colmar », Plos Biology, vol. 8, n 11, 2010. 


\section{Bibliographie}

Amable, Bruno et Stefano Palombarini, L'économie politique n'est pas une science morale, Paris, Raisons d'Agir Éditions, 2005.

Ancori, Bernard, "Analogie, évolution scientifique et réseaux complexes ", Nouvelles perspectives en sciences sociales. Revue internationale de systémique complexe et d'études relationnelles, vol. 1, n 1, 2005, p. 5-61.

Ancori, Bernard, "Analogie, métaphore et mutation féodale ", dans Sylvain David, Janusz Przychodzen et François-Emmanuel Boucher (dir.), Que peut la métaphore? Histoire, savoir et poétique, Paris, L'Harmattan, 2009, p. 115-131.

Ancori, Bernard, "Controverses et consensus scientifiques et sociotechniques entre théorie des consensus et sémantique des mondes possibles ou Glissements progressifs des sciences économiques vers la logique formelle. Petit exercice de réflexivité sous forme de témoignage sur une pratique de formalisation dans la construction d'une épistémologie ", Séminaire de l'Institut de Recherches Interdisciplinaires sur les Sciences et la Technologie, (IRIST EA 3424), Strasbourg, 24 novembre 2006.

Ancori, Bernard, Échange monétaire et Évolution économique, Thèse de Doctorat d'État ès-Sciences économiques, Université Louis Pasteur (Strasbourg I), 1990.

Ancori, Bernard, "Espace-temps d'un réseau sociocognitif complexe. I. Propension à communiquer et présent spécieux ", Nouvelles perspectives en sciences sociales. Revue internationale de systémique complexe et d'études relationnelles, vol. $3, \mathrm{n}^{\circ}$ 2, 2008, p. 113-181.

Ancori, Bernard, "Espace-temps d'un réseau sociocognitif complexe. II. Temporalités historiques et entropie sociocognitive ", Nouvelles perspectives en sciences sociales. Revue internationale de systémique complexe et d'études relationnelles, vol. 4, $\mathrm{n}^{\circ}$ 1, 2008, p. 9-76

Ancori, Bernard, "Évolution, complexité et consensus monétaire : un modèle théorique et quelques illustrations historiques ", Économie Appliquée, Tome L, n 3, 1997, p. 199-236.

Ancori, Bernard, «Expertise et citoyenneté : les Grecs anciens et nous. De l'Agora antique aux forums hybrides modernes ", Revue d'Anthropologie des Connaissances, vol. 3, n 3, Paris, 2009, p. 485-529.

Ancori, Bernard, "La production et la circulation des connaissances scientifiques et des savoirs profanes dans nos sociétés techniciennes ", dans François Darbellay (dir.), La circulation des savoirs. Interdisciplinarité, concepts nomades, analogies, métaphores, Berne, Éditions scientifiques internationales Peter Lang, 2012, p. 203-240. 
Ancori, Bernard, "Réseaux complexes et genèse des conventions ", $\sigma^{e}$ Congrès Européen des Sciences des Systèmes, Paris, 19-22 septembre 2005.

Bateson, Gregory, Steps to an Ecology of Mind, New York, Chandler Publishing Company, 1972.

Batifoulier, Philippe, Théorie des conventions, Paris, Economica, 2001.

Belarte, Bruno, Simulation de comportements sociaux dans un réseau d'agents individuels, Mémoire de TER, Fouille de Données et Bioinformatique Théorique du Laboratoire des Sciences de l'Image, de l'Informatique et de la Télédétection (FDBT-LSIIT, UMR 7002 CNRS-Université de Strasbourg), avril 2010.

Bensaude-Vincent, Bernadette, Les vertiges de la technoscience. Façonner le monde atome par atome, Paris, La Découverte, 2009.

Bensaude-Vincent, Bernadette et Dorothée Benoit-Browaeys, Fabriquer la vie. Où va la biologie de synthèse?, Paris, Seuil, 2011.

Besnier Jean-Michel, "Seul le désordre est créateur. Pour en finir avec les bataillons disciplinaires ", Hermès, n 67 , Paris, CNRS Éditions, 2013, p. 25-31.

Black Max, Models and Metaphors. Studies in Language and Philosophy, Ithaca, Cornell University Press, 1962.

Black Max, "More about Metaphor ", dans Andrew Ortony (dir.), Metaphor and Thought, Cambridge, Cambridge University Press, 1963, p. 19-41.

Boyer, Robert et André Orléan, "Persistance et changement des conventions. Deux modèles simples et quelques illustrations ", dans André Orléan (dir.), Analyse économique des conventions, Paris, Presses Universitaires de France, 1994, p. 219-247.

Brabandere, Luc de, Pensée magique, pensée logique. Petite philosophie de la créativité, Paris, Éditions du Pommier, 2012.

Breton, Philippe, «La représentation de l'humain et la construction d'une image de l'homme "sans intérieur" ", dans Dominique Weil (dir.), Figures du sujet dans la modernité, Strasbourg, Éditions Arcanes, 1997, p. 229-242.

Breton, Philippe et Serge Proulx, L'explosion de la communication, Paris, La Découverte, 1989.

Breton, Philippe et David Le Breton, Le silence et la parole. Contre les excès de la communication, Toulouse, Éditions Érès, 2009.

Calame, Matthieu, Lettre ouverte aux scientistes. Alternatives démocratiques à une idéologie cléricale, Paris, Éditions Charles Léopold Mayer, 2011. 
Callon, Michel, «Le réseau comme forme émergente et comme modalité de coordination : le cas des interactions stratégiques entre firmes industrielles er laboratoires académiques ", dans Michel Callon et al., Réseau et coordination, Paris, Economica, 1999, p. 13-64.

Callon, Michel et Yannick Barthe, "Décider sans trancher. Négociations et délibérations à l'heure de la démocratie dialogique ", Négociations, $\mathrm{n}^{\circ} 2$, 2005, p. 115-129.

Callon, Michel, Pierre Lascoume et Yannick Barthe, Agir dans un monde incertain. Essai sur la démocratie technique, Paris, Seuil, 2001.

Caron, François, La dynamique de linnovation. Changement technique et changement social (XVI $-X X^{e}$ siècle), Paris, Gallimard, 2010.

Caron, François, Les voies de l'innovation : les leçons de l'histoire, Paris, Éditions Manucius, coll. «Modélisations des imaginaires », 2011.

Castells, Manuel, "Ni dieu ni maître : les réseaux ", Fondation Maison des Sciences de l'Homme, Working Paper 02, février 2012.

Castells, Manuel, La société en réseaux. L'ère de l'information, Paris, Fayard, 1998.

Catellin, Sylvie et Laurent Loty, "Sérendipité et indisciplinarité ", Hermès, $n^{\circ}$ 67, Paris, CNRS Éditions, 2013, p. 32-40.

Conner, Clifford D., Histoire populaire des sciences, Paris, Éditions L'Échappée, 2011 [2005].

Coster, Michel de, L'analogie en sciences humaines, Paris, Presses Universitaires de France, 1978.

Degenne, Alain et Michel Forsé, "Comment on trouve ses amis. Enquête sur la sociabilité des Français ", dans Les liens sociaux invisibles, numéro hors série de Sciences Humaines, n 5, mai-juin 1994 p. 20-24.

Degenne, Alain et Michel Forsé, Les Réseaux sociaux, Paris, Armand Colin, 1994.

Détienne, Marcel, Les Maîtres de Vérité dans la Grèce archaïque, Paris, Maspéro, 1967.

Dogan, Mattei et Robert Pahre, L'innovation dans les sciences sociales. La marginalité créatrice, Paris, Presses Universitaires de France, 1991.

Durand-Richard, Marie-José et al., L'analogie dans la démarche scientifique. Perspective historique, Paris, L'Harmattan, 2008.

Gell-Mann, Murray, Le quark et le jaguar. Voyage au cour du simple et $d u$ complexe, Paris, Albin Michel, 1995 [1994].

Gineste, Marie-Dominique, Analogie et cognition. Étude expérimentale et simulation informatique, Paris, Presses Universitaires de France, 1997.

Goffman, Erving, Frame Analysis: An Essay on the Organization of Experience, New York, Harper \& Row, 1974. 
Gomez, Pierre-Yves, Qualité et Théorie des Conventions, Paris, Economica, 1994.

Halleux, Robert, Le savoir de la main. Savants et artisans dans l'Europe pré-industrielle, Paris, Armand Colin, 2009.

Hallyn, Fernand, Les structures rhétoriques de la science. De Képler à Maxwell, Paris, Seuil, 2004.

Hofstadter, Douglas et Emmanuel Sander, L'analogie, cæur de la pensée, Paris, Odile Jacob, 2013.

Jeanpierre, Laurent, "Une opposition structurante pour l'anthropologie structurale : Lévi-Strauss contre Gurvitch, la guerre de deux exilés français aux États-Unis ", Revue d'histoire des sciences humaines, $\mathrm{n}^{\circ} 11$, 2004, p. 13-43.

Koestler, Arthur, The Act of Creation, New York, Penguin Books, 1964.

Kuhn, Thomas S., The Structure of Scientific Revolutions, Chicago, University of Chicago Press, 1962.

Lafontaine, Cécile, L'empire cybernétique. Des machines à penser à la pensée machine, Paris, Seuil, 2004.

Lagasnerie, Geoffroy de, Logique de la création. Sur l'Université, la vie intellectuelle et les conditions de l'innovation, Paris, Fayard, 2011.

Lakoff, George et Mark Johnson, Metaphors We Live By, Chicago, The University of Chicago, 1980.

Lakoff, George et Mark Johnson, Philosophy in the Flesh. The Embodied Mind and Its Challenge to Western Thought, New York, Basic Books, 1999.

Larivière, Vincent., Éric Archambault et Yves Gingras, « Long-Term Variations in the Aging of Scientific Literature: From Exponential Growth to Staedy-State Science (1900-2004) ", Journal of the American Society for Information Science and Technology, vol. 59, $\mathrm{n}^{\circ}$ 2, 2008, p. 288-296.

Le Jallé, Éléonore, "La convention : ce que Lewis doit (ou non) à Hume ", Klesis - Revue philosophique, $\mathrm{n}^{\circ}$ 24, 2012, p. 239-271.

Le Ny, Jean-François, Comment l'esprit produit du sens. Notions et résultats des sciences cognitives, Paris, Odile Jacob, 2005.

Lévi-Strauss, Claude, L'anthropologie face aux problèmes du monde moderne, Paris, Seuil, 2011.

Lévy-Leblond, Jean-Marc, Le grand écart. La science entre technique et culture, Paris, Éditions Manucius, coll. "Modélisations des imaginaires ", 2013.

Lewis, David, Convention: A Philosophical Study, Cambridge (MA), Harvard University Press, 1969. 
Lewis, David K., "Languages and Language », Philosophical Papers, vol. I, Oxford University Press, 1983.

Lichnerowicz, André, François Perroux et Gilbert Gadoffre (dir.), Analogie et connaissance, Tome I - Aspects historiques, Paris, Éditions Maloine, 1980.

Lipinski, Marc, Les sciences, un enjeu citoyen. Une politique écologiste de la recherche et de l'innovation, Paris, Éditions Les petits matins, 2011.

Maffesoli, Michel, Le Temps des tribus. Le déclin de l'individualisme dans les sociétés de masse, Paris, Librairie des Méridiens, Klincksieck et Cie, 1988.

Maffesoli, Michel, La Transfiguration du Politique. La tribalisation du monde, Paris, Grasset et Fasquelle, 1992.

Maffesoli, Michel et Brice Perrier, L'homme postmoderne, Paris, François Bourin Éditeur, 2012.

Miller, Arthur I., Insights of Genius. Imagery and Creativity in Science and Art, New York, Springer Verlag Inc., 1996.

Moatti, Alexandre, Alterscience. Postures, dogmes, idéologies, Paris, Odile Jacob, 2013.

Moneyron Anne, Olivier Lemaire et Jean Masson, « Une expérience d'interface science/société. Consolider la recherche en s'appuyant sur les savoirs profanes, la complexité et l'engagement sur le temps long ", Futuribles, mars, n $^{\circ} 383,2012$, p. 111-118.

Naudon, Frédéric, "Comment le profane joue en faveur du décloisonnement ", Hermès, nº 67, CNRS Éditions, 2013, p. 62-67.

Orléan, André (dir.), Analyse économique des conventions, Paris, Presses Universitaires de France, 1994.

Ortony, Andrew (dir.), Metaphor and Thought, Cambridge, Cambridge University Press, 1993.

Parizeau, Marie-Hélène, Biotechnologie, nanotechnologie, écologie. Entre science et idéologie, Versailles, Éditions Quae, 2010.

Pestre, Dominique, À contre-science. Politiques et savoirs des sociétés contemporaines, Paris, Seuil, 2013.

Pestre, Dominique, Science, argent et politique. Un essai d'interprétation, Paris, INRA Éditions, 2003.

Richards, Ivor A., The Philosophy of Rhetoric, Oxford, Oxford University Press, 1936.

Rosenblueth, Arturo, Norbert Wiener et Julian Bigelow, "Behavior, Purpose and Teleology ", Philosophy of Science, vol. X, 1943, p. 18-24. 
Rosnay, Joël de, "L'impact des nouvelles technologies dans l'échange de savoir ", dans Savoir échanger les savoirs, Paris, Éditions Textuel, 1997, p. $55-74$.

Schlanger, Judith, "La pensée inventive », dans Judith Schlanger et Isabelle Stengers, Les concepts scientifiques : invention et pouvoir, Paris, La Découverte, 1989, p. 67-100.

Schelling, Thomas C., The Strategy of Conflict, Oxford, Oxford University Press, 1960.

Segal, Jérôme, Le Zéro et le Un. Histoire de la notion scientifique d'information au XXe siècle, Paris, Éditions Syllepse, 2003.

Sfez, Lucien, Critique de la communication, Paris, Seuil, 1989.

Smith, John Maynard, Evolution and the Theory of Games, Cambridge, Cambridge University Press, 1982.

Stengers, Isabelle, Une autre science est possible ! Manifeste pour un ralentissement des sciences, suivi de William James, Le poulpe du doctorat, présenté par Thierry Drumm, Paris, La Découverte, 2013.

The Local Monitoring Committee, "Interactive Technology Assessment and Beyond : the Field Trial of Genetically Modified Grapevines at INRA Colmar ", Plos Biology, vol. 8, n 11, 2010.

Triclot, Mathieu, Le moment cybernétique. La constitution de la notion d'information, Paris, Éditions Champ Vallon, 2008.

Wiener, Norbert, Cybernetics, or Control and Communication in the Animal and the Machine, Cambridge (MA), MIT Press, 1948.

Zwirn, Denis et Hervé Zwirn, «La révision des croyances ", Pour la science, $n^{\circ} 311$, septembre, 2003, p. 64-69. 